\title{
Generalized Quantum Stochastic Processes on Fock Space
}

\author{
By \\ Nobuaki OBATA* \\ Introduction
}

As is highlighted in the excellent books by Meyer [21] and by Parthasarathy [26] quantum stochastic calculus on (Boson) Fock space has developed into a new field of mathematics keeping a profound contact with physical applications. Since Hudson and Parthasarathy [12] first formulated quantum stochastic integrals of Itô type in 1984 a crucial role has been played by three basic quantum stochastic processes:

$$
A_{t}=\int_{0}^{t} \partial_{s} d s, \quad A_{t}^{*}=\int_{0}^{t} \partial_{i}^{*} d s, \quad \Lambda_{t}=\int_{0}^{t} \partial_{i}^{*} \partial_{1} d s, \quad t \geq 0
$$

where the notation is after our convention, see below. These are called the annihilation process, the creation process and the number (gauge) process, respectively. Afterwards there have appeared many developments and variants of approaches, among others see Belavkin [1], [2], [3], Lindsay [16], [17], LindsayMaassen [18], Maassen [19], Meyer [20] and Parthasarathy-Sinha [27].

In this paper we study another aspect of quantum stochastic processes on the basis of white noise calculus, which has been recently established as a Schwartz type distribution theory on Gaussian space [7], [8], [9], [15], with an interesting application to operator theory on Fock space [23]. In fact, in the operator theory a principal role has been played by an integral kernel operator

$$
\Xi_{l, m}(\kappa)=\int_{R^{\prime+m}} \kappa\left(s_{1}, \cdots, s_{l}, t_{1}, \cdots, t_{m}\right) \partial_{1_{1}}^{*} \cdots \partial_{s_{1}}^{*} \partial_{t_{1}} \cdots \partial_{t_{m}} d s_{1} \cdots d s_{l} d t_{1} \cdots d t_{m},
$$

where $\partial_{t}$ and $\partial_{t}^{*}$ are respectively the annihilation and creation operators at a point $t \in \mathbb{R}$, and $\kappa$ is a distribution in $l+m$ variables. For example, the three operators in $(0.1)$ are integral kernel operators of this type. Under the name of white noise calculus, we make a special choice of test and generalized functions on the Gaussian space $\left(E^{*}, \mu\right)$ and we work on a particular Gelfand triple

Communicated by H. Araki, October 6, 1994.

1991 Mathematics Subject Classification(s): 81 S25 46F25 47B38

* Graduate School of Polymathematics, Nagoya University, Nagoya 464-01, Japan 


$$
(E) \subset\left(L^{2}\right) \equiv L^{2}\left(E^{*}, \mu\right) \subset(E)^{*}
$$

where the middle space is a realization of the Fock space via the Wiener- ItôSegal theory. It is thus natural to discuss continuous operators between these spaces. In particular, $\partial_{t} \in \mathscr{L}^{\prime}((E),(E)), \partial_{t}^{*} \in \mathscr{L}^{\prime}\left((E)^{*},(E)^{*}\right)$ and $\Xi_{l, m}(\kappa) \in \mathscr{L}((E)$, $\left.(E)^{*}\right)$, which are important consequences of our approach. The integral kernel operator (0.2) was first formulated in [10], though similar expressions have appeared in various contexts, see e.g., [4], [5], [13], [21]; among others Belavkin [1] discusses norm estimates introducing an idea of Fock scale where a common spirit is observed.

The idea of integral kernel operators has developed into the theory of Fock expansion [23]. An important consequence is that every continuous operator in $\mathscr{Z}\left((E),(E)^{*}\right)$ admits an infinite series expansion in terms of integral kernel operators with a precise estimate of convergence. On the other hand, since $\kappa$ in

$(0.2)$ is considered as scalar-operator-valued distribution, it is natural to introduce an integral kernel operator of the form:

$$
\Xi=\int_{R^{\prime+m}} \partial_{s_{1}}^{*} \cdots \partial_{s_{1}}^{*} L\left(s_{1}, \cdots, s_{l}, t_{1}, \cdots, t_{m}\right) \partial_{t_{1}} \cdots \partial_{t_{m}} d s_{1} \cdots d s_{l} d t_{1} \cdots d t_{m},
$$

where $L$ is an $\mathcal{Z}\left((E),(E)^{*}\right)$-valued distribution on $\mathbb{R}^{1+m}$. This operator was first introduced in [25] to construct a quantum Hitsuda-Skorokhod integral. Moreover, Huang's idea of quantum stochastic measures [11] are included as a particular case. Thus we believe we are in a good position to discuss quantum stochastic processes along with the integral kernel operators and the theory of Fock expansion.

We briefly sketch the contents: In Section 1 we recapitulate some results in white noise calculus. The basic references are [23] and [24].

In Section 2 we propose the following

Definition. A family of operators $\left\{\Xi_{t} ; t \in \mathbb{R}\right\} \subset Y\left((E),(E)^{*}\right)$ is called a quantum stochastic process (on Fock space) if the map $t \mapsto \Xi_{t}$ is continuous. A continuous linear map $\Xi: E_{C} \rightarrow \downarrow\left((E),(E)^{*}\right)$ is called a generalized quantum stochastic process. A generalized quantum stochastic process $\Xi$ is called regular if it admits a continuous extension from $E_{C}^{*}$ into $\mathcal{f}\left((E),(E)^{*}\right)$. Here $E_{C}$ denotes the complexification of $\mathcal{Y}(\mathbb{R})$, the Schwartz space of rapidly decreasing functions on $\mathbb{R}$.

The continuity condition is not very strong because, for example, the white noise $\{x(t)\}$ becomes a continuous flow in $(E)^{*}$ within the white noise setup. Hence, the quantum white noise, i.e., the white noise regarded as multiplication operators, is a (in fact, regular) quantum stochastic process in our sense. In this section we discuss some fundamental properties of quantum stochastic processes and obtain the Fock expansions. 
Section 3 is devoted to a study of quantum stochastic integrals without assuming the adaptedness. For a quantum stochastic process $\left\{L_{t}\right\}$ the operatorvalued integral

$$
\Xi_{t}=\int_{a}^{t} L_{s} d s
$$

is defined by a standard method of functional analysis and again becomes a quantum stochastic process. Since $\left\{L_{1} \partial_{1}\right\}$ and $\left\{\partial_{,}^{*} L_{1}\right\}$ are also quantum stochastic processes, we naturally obtain new ones:

$$
\int_{a}^{t} L, \partial, d s, \quad \quad \quad \int_{a}^{t} \partial^{*} L, d s .
$$

These are called the quantum stochastic integrals of $\left\{L_{t}\right\}$ against the annihilation process and the creation process, respectively. The latter generalizes a quantum Hitsuda-Skorokhod integral discussed in [16], [17], [25]. By Riemannian approximation, we obtain

$$
\int_{a}^{t} L_{s} \partial_{,} d s=\int_{a}^{t} L_{a} d A_{s}
$$

where the right hand side is a natural extension of the quantum stochastic integral of Itô type due to Hudson-Parthasarathy [12] to cover the non-adapted case.

In Section 4 we introduce the concept of an adapted process by using the commutativity with $\partial_{t}$ and $\partial_{t}^{*}$. Our definition is compatible with those due to Hudson-Parthasarathy [12], Huang [11] and others. Then for an adapted process $\{L\}$ we have

$$
\int_{a}^{t} \partial^{*} L_{a} d s=\int_{a}^{t} L_{1} d A^{*}
$$

where the right hand side is defined through the Riemannian approximation and coincides with a stochastic integral of Itô type introduced in [12]. Moreover, we derive a stochastic integral representation of an adapted process. By way of illustration assume that $\Xi$ is an adapted and regular generalized quantum stochastic process. Then it is expressed in the form:

$$
\Xi_{t}=\int_{-\infty}^{t} L(t, s) \partial_{1} d s+\int_{-\infty}^{t} \partial^{*} M^{*}(t, s) d s+c_{t} I, \quad t \in \mathbb{R}
$$

where

(i) $\quad L: \mathbb{R} \times E_{\boldsymbol{C}} \rightarrow /\left((E),(E)^{*}\right)$ is continuous; linear in the second argument; and $\operatorname{supp} L(t, \cdot) \subset(-\infty, t]$ for all $t \in \mathbb{R}$;

(ii) $\quad M: R \times E_{C} \rightarrow \swarrow((E),(E))$ is continuous; linear in the second argument; $\left[M(s, \xi), \partial_{t}\right]=0$ for any $\xi \in E_{C}$ and $s, t \in \mathbb{R}$; and $\operatorname{supp} M(t, \cdot) \subset(-\infty, t]$ for all $t \in \boldsymbol{R}$

(iii) $c: \boldsymbol{R} \rightarrow \boldsymbol{C}$ is a continuous function. 
For more details see $\$ 4.3$. It is remarkable to have such standard expression as in (0.4) and we may expect further applications, for example, to quantum martingales [27].

General Notation. Let $\mathfrak{X}, \mathfrak{P}), \mathfrak{i})$ be locally convex spaces.

$Y(\mathfrak{X}, \mathfrak{Y})$ ): the space of continuous linear operators from $\mathfrak{X}$ into $\mathfrak{Y})$; equipped with the topology of bounded convergence.

$\mathscr{B}(\mathfrak{X}, \mathfrak{Y} ; \mathfrak{3})$ : the space of continuous bilinear maps from $\mathfrak{X} \times \mathfrak{Y}$ into $\mathfrak{Z}$; equipped with the topology of bi-bounded convergence.

$\mathscr{\mathscr { s }}_{\text {sep }}(\mathfrak{X}, \mathfrak{Y} ; ; \mathfrak{Z})$ : the space of separately continuous bilinear maps from $\mathfrak{X} \times \mathfrak{V}$ into 3 ; no topology is needed.

$\mathfrak{X}^{*}$ : the space of continuous linear functionals on $\mathfrak{X}$; equipped with the strong dual topology after our convention above.

$\mathfrak{X} \otimes \mathfrak{Y}$ : the Hilbert space tensor product when both $\mathfrak{X}, \mathfrak{P}$ ) are Hilbert spaces.

$\mathfrak{X} \otimes_{\pi} \mathfrak{V}:$ the completed $\pi$-tensor product. When there is no danger of confusion, $\otimes_{\pi}$ is denoted by $\otimes$ for simplicity.

\section{$\S 1$. Operators on Fock Space}

\section{$\S$ H。目。White Noise Triplet}

Throughout let $H$ denote the Hilbert space of $\mathbb{R}$-valued square integrable functions on the real line $\mathbb{R}$ with respect to the Lebesgue measure $d t$, where the real line is considered as the time axis. The norm and inner product are denoted by $|\cdot|=|\cdot|_{0}$ and $\langle\cdot, \cdot\rangle$, respectively. These are naturally extended to the norm and the $C$-bilinear form on $H_{C}$, the complexification of $H$. Note therefore that $\langle\cdot, \cdot\rangle$ is not a Hermitian inner product of $H_{C}$.

In order to realize the Fock space over $H_{C}$ we adopt a particular Gelfand triple:

$$
E=\text { ソ }(\mathbb{R}) \subset H=L^{2}(\mathbb{R}) \subset E^{*}=\mathcal{J}^{\prime}(\mathbb{R}),
$$

where $\mathcal{f}(\mathbb{R})$ is the space of rapidly decreasing functions and $\mathcal{Y}^{\prime}(\mathbb{R})$ its dual space, i.e., the space of tempered distributions. The canonical bilinear form on $E^{*} \times E$ is also denoted by $\langle\cdot, \cdot\rangle$. The Gaussian measure $\mu$ is by definition the unique probability measure on $E^{*}$ of which the characteristic function is:

$$
\exp \left(-\frac{1}{2}|\xi|_{0}^{2}\right)=\int_{E} e^{\iota\langle 1 . \xi\rangle} \mu(d x), \quad \xi \in E
$$

The probability space $\left(E^{*}, \mu\right)$ is called the Gaussian space. Let $\left(L^{2}\right)=L^{2}\left(E^{*}, \mu\right)$ be the Hilbert space of $\mathbb{C}$-valued square-integrable functions on $E^{*}$ with respect to the Gaussian measure $\mu$. The norm is denoted by $\|\cdot\|_{0}$. 
The Wiener-Itô-Segal theory says that $\left(L^{2}\right)$ is canonically isomorphic to the Fock space over $H_{C}$. To be more precise, we introduce notation. The canonical bilinear form on $\left(E^{\otimes n}\right)^{*} \times\left(E^{\otimes_{n}}\right)$ is denoted by $\langle\cdot, \cdot\rangle$ again and its $C$-bilinear extension to $\left(E_{C}^{\otimes_{n}}\right)^{*} \times\left(E_{C}^{\otimes_{n}}\right)$ is also denoted by the same symbol. The "renormalized" tensor product $: x^{\otimes n}: \in\left(E_{C}^{\otimes n}\right)_{\text {sym }}^{*}$ is uniquely determined by the exponential vector:

$$
\phi_{\xi}(x) \equiv \exp \left(\langle x, \xi\rangle-\frac{1}{2}\langle\xi, \xi\rangle\right)=\sum_{n=0}^{\infty}\left\langle: x^{\otimes n}: \frac{\xi^{\otimes n}}{n !}\right\rangle, \quad \xi \in E_{C} .
$$

Here, in particular, $\phi_{0}$ is called the vacuum. With these notation every $\phi \in\left(L^{2}\right)$ admits the Wiener-Ito expansion:

$$
\phi(x)=\sum_{n=0}^{\infty}\left\langle: x^{\otimes n}:, f_{n}\right\rangle, \quad x \in E^{*}, \quad f_{n} \in H_{C}^{\otimes} \hat{\otimes}^{\prime} .
$$

In that case it holds that

$$
\|\phi\|_{0}^{2} \equiv \int_{E}|\phi(x)|^{2} \mu(d x)=\sum_{n=0}^{\infty} n !\left|f_{n}\right|_{0}^{2} .
$$

We next construct a sort of Fock space over $E_{C}$. Recall that the topology of $E$ is given by the (semi)norms:

$$
|\xi|_{p}=\left|A^{p} \xi\right|_{0}, \quad \xi \in E, \quad p \in \mathbb{R}
$$

where $A=1+t^{2}-d^{2} / d t^{2}$. The constant numbers $0<\rho=\left\|A^{-1}\right\|_{O P}<1$ and $\delta=\left\|A^{-1}\right\|_{H S}$ $<\infty$ are frequently used throughout. In fact, $\rho=1 / 2$ and $\delta=\pi / \sqrt{24}$. Suppose that the Wiener-Itô expansion of $\phi \in\left(L^{2}\right)$ is given as in (1.1). Taking (1.2) into account, we put

$$
\|\phi\|_{p}^{2}=\sum_{n=0}^{\infty} n !\left|f_{n}\right|_{p}^{2}, \quad p \in \mathbb{R}
$$

Let $(E)$ be the space of all $\phi \in\left(L^{2}\right)$ such that $\|\phi\|_{p}<\infty$ for all $p \in \mathbb{R}$. Then $(E)$ becomes a countable Hilbert nuclear space with norms $\|\cdot\|_{p}, p \in \mathbb{R}$. For $p \in \mathbb{R}$ let $(E)_{p}$ denote the completion of $(E)$ with respect to the norm $\|\cdot\|_{p}$. Then $\left\{(E)_{p} ; p \in \mathbb{R}\right\}$ constitutes a chain of Hilbert spaces in both directions and we have

$$
\begin{aligned}
& (E) \cong \underset{p \rightarrow \infty}{\operatorname{proj} \lim }(E)_{p}\left(=\bigcap_{p \geq 0}(E)_{p} \text { as vector spaces }\right) \text {. } \\
& (E)^{*} \cong \operatorname{ind} \lim _{p \rightarrow \infty}(E)_{-p}\left(=\bigcup_{p \geq 0}(E)_{p} \text { as vector spaces }\right) .
\end{aligned}
$$

Finally we obtain a complex Gelfand triple:

$$
(E) \subset\left(L^{2}\right)=L^{2}\left(E^{*}, \mu ; C\right) \subset(E)^{*} .
$$


Elements in $(E)$ and $(E)^{*}$ are called a test (white noise) functional and a generalized (white noise) functional, respectively. We denote by $《 \cdot, \cdot\rangle$ the canonical bilinear form on $(E)^{*} \times(E)$.

For $\Phi \in(E)^{*}$ there exists a unique sequence $F_{n} \in\left(E_{C}^{\otimes n}\right)_{\text {sym }}^{*}$ such that $\|\Phi\|_{-p}^{2}=$ $\sum_{n=0}^{\infty} n !\left|F_{n}\right|_{-p}^{2}$ and

$$
\langle\Phi, \phi\rangle=\sum_{n=0}^{\infty} n !\left\langle F_{n}, f_{n}\right\rangle
$$

for $\phi \in(E)$ of which Wiener-Itô expansion is given as in (1.1). In that case we adopt a formal expression:

$$
\Phi(x)=\sum_{n=0}^{\infty}\left\langle: x^{\otimes n}:, F_{n}\right\rangle .
$$

This is also called the Wiener-Itô expansion of $\Phi$.

\section{$\S 1.2$. Spaces of Continuous Operators}

The Gelfand triple (1.3) suggests $\mathscr{L}^{\prime}\left((E),(E)^{*}\right)$ and $\mathscr{L}^{\prime}((E),(E))$ as natural classes of operators to be discussed. By definition their topologies are given by the seminorms:

$$
\begin{aligned}
\|\Xi\|_{B_{1}, B_{2}} & =\sup _{\phi \in B_{1}, \psi \in B_{2}}|\mathbb{Z} \Xi \phi, \psi\rangle \mid, \quad \Xi \in \mathcal{Z}\left((E),(E)^{*}\right), \\
\|\Xi\|_{B, p} & =\sup _{\phi \in B}\|\Xi \phi\|_{p}, \quad \Xi \in \mathcal{Y}^{\prime}((E),(E)),
\end{aligned}
$$

where $B_{1}, B_{2}, B$ run over the bounded subsets of $(E)$, and $p$ runs over $\mathbb{R}$ (or equivalently $p \geq 0)$. The natural injection: $\quad \mathcal{Z}((E),(E)) \rightarrow \mathscr{Z}\left((E),(E)^{* *}\right)$ is continuous by definition.

Since $(E)$ is reflexive, the adjoint operator of $\Xi \in \mathscr{L}^{\prime}\left((E),(E)^{*}\right)$, denoted by $\Xi^{*}$, again belongs to $\mathcal{Y}\left((E),(E)^{*}\right)$. The relation is given as

$$
\left\langle\Xi \phi, \psi 》=\left\langle\Xi^{*} \psi, \phi 》, \quad \phi . \psi \in(E)\right. \text {. }\right.
$$

Here one should note that $\langle\cdot \cdot \cdot\rangle$ is the canonical $\boldsymbol{C}$-bilinear form on $(E)^{*} \times(E)$.

Lemma 1.1. The map $\Xi \mapsto \Xi^{*}$ is a linear homeomorphism (i) from $\mathcal{Z}\left((E),(E)^{*}\right)$ onto itself; (ii) from $\mathcal{L}\left((E),(E)\right.$ ) onto $\mathcal{Y}\left((E)^{*},(E)^{* *}\right)$; (iii) from $\mathcal{Z}\left((E)^{*},(E)^{* *}\right)$ onto $\mathcal{\perp}((E),(E))$.

In the above statement, according to our convention, $f\left((E)^{*},(E)^{*}\right)$ is equipped with the topology of bounded convergence. The proof is straightforward. Note also that $\mathscr{L}\left((E)^{*},(E)^{*}\right)$ is identified with the subspace of operators in $\mathcal{L}\left((E),(E)^{*}\right)$ which are continuously extended to those from $(E)^{*}$ into itself. By the above lemma we do not need special care for $Y\left((E)^{*},(E)^{*}\right)$. 
Recall the canonical isomorphism:

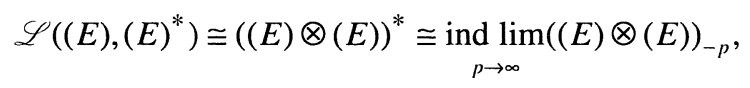

which follows from the kernel theorem (see also [23]). Then, for $p \geq 0$ let $\mathscr{Z}_{p}\left((E),(E)^{*}\right)$ denote the subspace of all $\Xi \in \mathscr{L}^{\prime}\left((E),(E)^{*}\right)$ which correspond to elements in $((E) \otimes(E))_{-p}$. The topology of $\mathscr{L}_{p}\left((E),(E)^{*}\right)$ is naturally induced from the norm of $((E) \otimes(E))_{-p}$ which is denoted by $\|\cdot\|_{-p}$. With this notation,

$$
\|\Xi \phi\|_{-p} \leq\|\Xi\|_{-p}\|\phi\|_{p}, \quad \phi \in(E) .
$$

Note also that

$$
\mathscr{L}^{\prime}\left((E),(E)^{*}\right) \cong \text { ind } \lim _{p \rightarrow \infty} \mathscr{L}_{p}^{\prime}\left((E),(E)^{*}\right)\left(=\bigcup_{p \geq 0} \mathscr{L}_{p}\left((E),(E)^{*}\right) \text { as vector spaces }\right),
$$

In particular, $\mathscr{L}_{0}\left((E),(E)^{*}\right)$ is identified with the space of all Hilbert-Schmidt operators on $\left(L^{2}\right)$.

Remark. By (1.5) there is a natural injection from $\mathscr{Z}_{p}\left((E),(E)^{*}\right)$ into $\mathcal{Z}\left((E)_{p},(E)_{-p}\right)$, where $\mathcal{Z}\left((E)_{p},(E)_{-p}\right)$ is identified with the subspace of all operators $\Xi \in \mathcal{L}\left((E),(E)^{*}\right)$ which admit continuous extensions from $(E)_{p}$ into $(E)_{-p}$. Note that the injection is not surjective. In fact, we have

$$
\mathscr{L}^{\prime}\left((E)_{p},(E)_{-p}\right) \cong \mathscr{B}\left((E)_{p},(E)_{p}\right) \cong\left((E)_{p} \otimes_{\pi}(E)_{p}\right)^{*}
$$

and

$$
\mathscr{Y}_{p}^{\prime}\left((E),(E)^{*}\right) \cong((E) \otimes(E))_{-p} \cong\left((E)_{p} \otimes(E)_{p}\right)^{*} .
$$

Thus very crucial is the difference between the $\pi$ - and the Hilbert space tensor products of Hilbert spaces. On the other hand, by a general result on a countable Hilbert space we have

$$
\mathscr{Y}\left((E),(E)^{*}\right)=\bigcup_{p \geq 0} \mathscr{Y}\left((E)_{p},(E)_{-p}\right)
$$

In particular, all bounded operators on the Fock space $\left(L^{2}\right)=(E)_{0}$ belong to $\mathcal{L}^{\prime}\left((E),(E)^{*}\right)$.

In general, the image of a bounded subset under $\Xi \in \mathcal{Z}((E),(E))$ is again a bounded subset.

Lemma 1.2. For $\Xi_{1} \in \mathcal{Y}\left((E),(E)^{*}\right)$ and $\Xi_{2} \in \mathcal{Z}((E),(E))$

$$
\begin{aligned}
& \left\|\Xi_{1} \Xi_{2}\right\|_{B_{1}, B_{2}} \leq\left\|\Xi_{1}\right\|_{-p}\left\|\Xi_{2}\right\|_{B_{1}, p}\left\|B_{2}\right\|_{p} \\
& \left\|\Xi_{1} \Xi_{2}\right\|_{B_{1}, B_{2}}=\left\|\Xi_{1}\right\|_{\Xi_{2} B_{1}, B_{2}}
\end{aligned}
$$


where $B_{1}, B_{2} \subset(E)$ are bounded subsets and $p \geq 0$. If $\Xi_{1}, \Xi_{2} \in \mathcal{Z}((E),(E))$, then

$$
\left\|\Xi_{1} \Xi_{2}\right\|_{B . p}=\left\|\Xi_{1}\right\|_{\Xi_{2} B, p},
$$

where $B \subset(E)$ is a bounded subset and $p \geq 0$.

Proof. Suppose $\Xi_{1} \in \mathcal{Z}\left((E),(E)^{*}\right)$ and $\Xi_{2} \in \mathcal{Z}((E),(E))$. Then by definition,

$$
\begin{aligned}
\left\|\Xi_{1} \Xi_{2}\right\|_{B_{1}, B_{2}} & =\sup _{\phi \in B_{1}, \psi \in B_{2}}\left|\left\langle\Xi_{1} \Xi_{2} \phi, \psi\right\rangle\right|=\sup _{\phi \in B_{1}, \psi \in B_{2}}\left|\mathbb{\Xi _ { 2 }}, \Xi_{1}, \phi \otimes \psi\right\rangle \mid \\
& \leq \sup _{\phi \in B_{1}, \psi \in B_{2}}\left\|\Xi_{1}\right\|_{-p}\left\|\Xi_{2} \phi\right\|_{p}\|\psi\|_{p}=\left\|\Xi_{1}\right\|_{-p}\left\|\Xi_{2}\right\|_{B_{1}, p}\left\|B_{2}\right\|_{p},
\end{aligned}
$$

which proves (1.6). The proofs of (1.7) and (1.8) are similar.

qed

\section{Proposition 1.3. The bilinear maps}

$$
\begin{aligned}
& \mathscr{L}^{\prime}\left((E),(E)^{*}\right) \times \mathscr{L}((E),(E)) \rightarrow \mathcal{L}^{*}\left((E),(E)^{*}\right) \\
& \mathscr{L}^{\prime}((E),(E)) \times \mathscr{L}^{*}((E),(E)) \rightarrow \mathscr{L}^{\prime}((E),(E))
\end{aligned}
$$

defined by the composition of operators are separately continuous.

Proof. For a fixed $\Xi_{1} \in \mathscr{L}^{\prime}\left((E),(E)^{*}\right)$ take $p \geq 0$ such that $\left\|\Xi_{1}\right\|_{-p}<\infty$. Then it follows from (1.6) that $\Xi_{2} \mapsto \Xi_{1} \Xi_{2}$ is a continuous linear map from $\mathscr{L}^{\prime}((E),(E))$ into $\mathscr{Z}^{\prime}\left((E),(E)^{*}\right)$. Next let $\Xi_{2} \in \mathcal{Z}((E),(E))$ be fixed. Then we see from (1.7) that $\Xi_{1} \mapsto \Xi_{1} \Xi_{2}$ is a continuous linear map from $\mathcal{Z}\left((E),(E)^{* *}\right)$ into $\mathcal{Z}\left((E),(E)^{*}\right)$. Thus the bilinear form (1.9) is separately continuous. As for (1.10) we need only to apply (1.8) and the fact that the natural injection $\mathcal{Y}((E),(E)) \rightarrow \mathcal{Z}\left((E),(E)^{*}\right)$ is continuous.

qed

\section{Corollary 1.4. The bilinear maps}

$$
\begin{aligned}
& \mathcal{Z}\left((E)^{*},(E)^{*}\right) \times \mathcal{Z}\left((E),(E)^{*}\right) \rightarrow \mathcal{Y}\left((E),(E)^{*}\right) \\
& \mathscr{L}^{*}\left((E)^{*},(E)^{*}\right) \times \mathcal{Y}\left((E)^{*},(E)^{*}\right) \rightarrow \mathcal{I}\left((E)^{*},(E)^{*}\right) .
\end{aligned}
$$

defined by the composition of operators are separately continuous.

Proof. Immediate from Proposition 1.3 by duality.

qed

Corollary $\mathbb{1} .5$. The composition of operators $\left(\Xi_{1}, \Xi_{2}\right) \mapsto \Xi_{1} \Xi_{2}$ is a (jointly) continuous bilinear map from $\left.\mathcal{L}_{p}\left((E),(E)^{*}\right) \times \mathcal{L}((E),(E)) \rightarrow \mathcal{L}_{(}(E),(E)^{*}\right)$ for a fixed $p \geq 0$.

Proof. Immediate from (1.6). 


\section{§1.3. Integral Kernel Operators}

We first recall creation and annihilation operators at a point $t \in \boldsymbol{R}$. Let $\phi \in(E)$ be given with Wiener- Itô expansion:

$$
\phi(x)=\sum_{n=0}^{\infty}\left\langle: x^{\otimes n}:, f_{n}\right\rangle, \quad f_{n} \in E_{C}^{\hat{\otimes}_{n}} .
$$

For any $y \in E_{C}^{*}$ we put

$$
D_{1} \phi(x)=\sum_{n=1}^{\infty} n\left\langle: x^{\otimes(n-1)}:, y \otimes_{1} f_{n}\right\rangle
$$

where $\otimes_{1}$ is the contraction of tensor products, see the end of this section. It is known that $D_{1} \in \mathcal{f}((E),(E))$ and hence $D_{1}^{*} \in \mathcal{Y}\left((E)^{*},(E)^{*}\right)$. For $\Phi \in(E)^{*}$ of which Wiener- Itô expansion is given as

$$
\Phi(x)=\sum_{n=0}^{\infty}\left\langle: x^{\otimes n}:, F_{n}\right\rangle, \quad F_{n} \in\left(E_{C}^{\otimes^{n}}\right)_{\mathrm{4ym}}^{*},
$$

we have

$$
D_{1}^{*} \Phi(x)=\sum_{n=0}^{\infty}\left\langle: x^{\otimes(n+1)}:, y \otimes F_{n}\right\rangle
$$

Since the delta functions $\delta_{t}$, belong to $E^{*}=. \mathcal{J}^{\prime}(\boldsymbol{R})$, we may define

$$
\partial_{t}=D_{\delta_{1}}, \quad t \in \mathbb{R}
$$

Then $\partial_{t} \in \mathcal{Y}((E),(E))$ and $\partial_{t}^{*} \in \mathcal{Y}\left((E)^{*},(E)^{*}\right)$ are respectively called the annihilation operator and the creation operator at a point $t \in \boldsymbol{R}$. Here we emphasize that these are not operator-valued distributions but continuous operators for themselves. The annihilation operator $\partial_{t}$ is also called Hida's differential operator.

For $\phi, \psi \in(E)$ consider a function on $\boldsymbol{R}^{1+m}$ defined by

$$
\eta_{\phi, \psi}\left(s_{1}, \cdots, s_{l}, t_{1}, \cdots, t_{m}\right)=\left\langle\partial_{s_{1}}^{*} \cdots \partial_{s_{1}}^{*} \partial_{t_{1}} \cdots \partial_{t_{m}} \phi, \psi\right\rangle .
$$

It is known that $\eta_{\phi . \psi} \in E_{C}^{\otimes(I+m)}$ and $\phi, \psi \mapsto\left\langle\kappa, \eta_{\phi . \psi}\right\rangle$ becomes a continuous bilinear form on $(E)$ for any $\kappa \in\left(E_{C}^{\otimes(I+m)}\right)^{*}$. Then by general theory there exists a unique continuous linear operator $\Xi_{l, m}(\kappa) \in \mathcal{Y}\left((E),(E)^{\prime \prime)}\right)$ such that

$$
\left\langle\Xi_{l, m}(\kappa) \phi, \psi\right\rangle=\left\langle\kappa, \eta_{\phi, \psi}\right\rangle, \quad \phi, \psi \in(E) .
$$

We employ a formal integral expression:

$$
\Xi_{l . m}(\kappa)=\int_{R^{++m}} \kappa\left(s_{1}, \cdots, s_{l}, t_{1}, \cdots, t_{m}\right) \partial_{t_{1}}^{*} \cdots \partial_{v_{1}}^{*} \partial_{t_{1}} \cdots \partial_{t_{m}} d s_{1} \cdots d s_{l} d t_{1} \cdots d t_{m}
$$

and call it an integral kernel operator with kernel distribution $\kappa$. The kernel 
distribution is unique if it is taken from the subspace

$$
\left(E_{C}^{\otimes(I+m)}\right)_{\mathrm{sym}(l, m)}^{*}=\left\{\kappa \in\left(E_{C}^{\otimes(I+m)}\right)^{*} ; s_{l, m}(\kappa)=\kappa\right\},
$$

where $s_{l, m}$ is the symmetrizing operator with respect to the first $l$ and the last $m$ variables independently. This is related to the fact that $\left[\partial_{s}, \partial_{t}\right]=0$ and $\left[\partial_{,}^{*}, \partial_{t}^{*}\right]=0$.

The most basic examples of integral kernel operators are annihilation and creation operators:

$$
D_{1}=\Xi_{0.1}(y)=\int_{R} y(t) \partial_{t} d t, \quad D_{v}^{*}=\Xi_{1,0}(y)=\int_{R} y(s) \partial_{,}^{*} d s, \quad y \in E_{C}^{*} .
$$

In particular, $\partial_{t}=\Xi_{0,1}\left(\delta_{t}\right)$ and $\partial_{t}^{*}=\Xi_{1,0}\left(\delta_{t}\right), \quad t \in \boldsymbol{R}$.

Theorem 1.6. Let $\kappa \in\left(E_{C}^{\otimes(l+m)}\right)^{*}$. Then $\Xi_{l, m}(\kappa) \in \mathcal{Z}^{\prime}((E),(E))$ if and only if $\kappa \in\left(E_{C}^{\otimes l}\right) \otimes\left(E_{C}^{\otimes m}\right)^{*}$. In particular, $\Xi_{0, m}(\kappa) \in \mathscr{L}((E),(E))$ for any $\kappa \in\left(E_{C}^{\otimes m}\right)^{* *}$.

The above result was proved in [10]. Moreover, we have

Proposition 1.7. The following commutative diagram holds:

$$
\begin{array}{ccc}
\left(E_{C}^{\otimes l}\right) \otimes\left(E_{C}^{\otimes m}\right)^{*} & \rightarrow & \left(E_{C}^{\otimes l} \otimes E_{C}^{\otimes m}\right)^{*} \\
\downarrow & & \downarrow \\
\mathcal{Z}^{\prime}((E),(E)) & \rightarrow & \mathscr{I}^{\prime}\left((E),(E)^{*}\right)
\end{array}
$$

where all arrows are continuous.

Proof. By a general theory (see e.g., [28]) one can prove

$$
\left(E_{C}^{\otimes I}\right) \otimes\left(E_{C}^{\otimes m}\right)^{*} \cong \underset{p \rightarrow \infty}{\operatorname{ind} \lim }\left(E_{C}^{\otimes I}\right) \otimes\left(E_{C}^{\otimes m}\right)_{-p} .
$$

Hence a linear map from $\left(E_{C}^{\otimes I}\right) \otimes\left(E_{C}^{\otimes m}\right)^{*}$ into $\mathscr{L}((E),(E))$ is continuous if and only if the restriction to $\left(E_{C}^{\otimes I}\right) \otimes\left(E_{C}^{\otimes m}\right)_{-p}$ is continuous. This follows from the norm estimates of $\Xi$, see Theorem A.1 in Appendix A.1. The rest of the assertion is already clear.

qed

It is possible to replace the kernel distribution $\kappa$ in an integral kernel operator (1.13) with an operator-valued distribution, for generalities for such distributions see [24]. With each $L \in \mathcal{Z}\left(E_{C}^{\otimes(l+m)}, \mathcal{Z}\left((E),(E)^{*}\right)\right)$ we may associate an operator $\Xi \in \mathcal{Y}\left((E),(E)^{*}\right)$ by the formula:

$$
《 \Xi \phi_{\xi}, \phi_{\eta} 》=\left\langle L\left(\eta^{\otimes /} \otimes \xi^{\otimes m}\right) \phi_{\xi}, \phi_{\eta} 》, \quad \xi, \eta \in E_{C} .\right.
$$

That $\Xi$ is well defined is due to the characterization theorem of operator symbols, see [23] and [25]. It is reasonable to write

$$
\Xi=\int_{R^{\prime+m}} \partial_{v_{1}}^{*} \cdots \partial_{v_{1}}^{*} L\left(s_{1}, \cdots, s_{l}, t_{1}, \cdots, t_{m}\right) \partial_{t_{1}} \cdots \partial_{t_{m}} d s_{1} \cdots d s_{l} d t_{1} \cdots d t_{m}
$$


In fact, if $L$ is a scalar-operator-valued distribution (1.15) is reduced to an integral kernel operator as in (1.13). The next result was shown in [25].

Theorem 1.8 (Fubini Type). Fix integers $0 \leq \alpha \leq l$ and $0 \leq \beta \leq m$. Given $\kappa \in\left(E_{C}^{\otimes(I+m)}\right)^{*}$ there exists $L \in \mathcal{Y}\left(E_{C}^{\otimes(\alpha+\beta)}, \mathcal{Z}\left((E),(E)^{*}\right)\right)$ such that

$$
\begin{aligned}
& L\left(\eta_{1} \otimes \cdots \otimes \eta_{\alpha} \otimes \xi_{1} \otimes \cdots \otimes \xi_{\beta}\right) \\
& =\Xi_{l-\alpha, m-\beta}\left(\left(\kappa \otimes_{\beta}\left(\xi_{1} \otimes \cdots \otimes \xi_{\beta}\right)\right) \otimes^{\alpha}\left(\eta_{1} \otimes \cdots \otimes \eta_{\alpha}\right)\right),
\end{aligned}
$$

where $\eta_{1}, \cdots, \eta_{\alpha}, \xi_{1}, \cdots, \xi_{\beta} \in E_{C}$. In that case

$$
\Xi_{l, m}(\kappa)=\int_{R^{\alpha+\beta}} \partial_{\uparrow_{1}}^{*} \cdots \partial_{\uparrow_{\alpha}}^{*} L\left(s_{1}, \cdots, s_{\alpha}, t_{1}, \cdots, t_{\beta}\right) \partial_{t_{1}} \cdots \partial_{t_{\beta}} d s_{1} \cdots d s_{\alpha} d t_{1} \cdots d t_{\beta} .
$$

To be sure, we recall the contraction of tensor products. Let $\kappa \in\left(E_{C}^{\otimes(l+m)}\right)^{*}$. For $g_{l} \in E_{C}^{\otimes l}$ and $g_{n} \in E_{C}^{\otimes n}$ we define $\kappa \otimes^{l}\left(g_{l} \otimes g_{n}\right) \in\left(E_{C}^{\otimes(m+n)}\right)^{*}$ as a unique element satisfying

$$
\left\langle\kappa \otimes{ }^{\prime}\left(g_{l} \otimes g_{n}\right), \zeta\right\rangle=\left\langle\kappa \otimes g_{n}, g_{l} \otimes \zeta\right\rangle, \quad \zeta \in E_{C}^{\otimes(m+n)} .
$$

Then $\kappa \otimes^{\prime} g$ is defined for any $g \in E_{C}^{\otimes(1+m)}$ by continuity and is called a left contraction. Moreover, it is easily verified that

$$
\left|F \otimes^{\prime} g\right|_{-p} \leq \rho^{2 m}|F|_{-p}|g|_{p}, \quad F \in\left(E_{C}^{\otimes(l+m)}\right)^{*}, \quad g \in E_{C}^{\otimes(l+n)} .
$$

The right contraction $\kappa \otimes, g$ is similar, for details see [23, $\$ 4.3]$.

\section{§2. Quantum Stochastic Processes}

\section{§2.1. Definition}

For each $t \in \boldsymbol{R}$ a generalized white noise functional $\Phi_{t}$ is defined by

$$
\Phi_{t}(x)=\left\langle: x:, \delta_{t}\right\rangle=\left\langle x, \delta_{t}\right\rangle, \quad t \in \boldsymbol{R} .
$$

In fact, since $\delta_{t} \in E^{*}=\gamma^{\prime}(\boldsymbol{R})$ for all $t \in \boldsymbol{R}$, we only need to follow the argument at the end of $\S 1.1$. For simplicity we write $x(t)=\Phi_{t}(x)$. The family $\{x(t)\} \subset(E)^{*}$, regarded as a "generalized" stochastic process, is called the white noise. Among many mathematical formulations of white noise, here is a noteworthy feature of our approach; namely, $t \mapsto \Phi$, is a continuous flow in $(E)^{*}$. This motivates us to make the following

Definition 2.1. A family of operators $\left\{\Xi_{t} ; t \in \boldsymbol{R}\right\} \subset Y\left((E),(E)^{*}\right)$ is called a quantum stochastic process (on Fock space) if the map $t \mapsto \Xi_{t}$ is continuous. A continuous linear map $\Xi: E_{C} \rightarrow \mathscr{L}\left((E),(E)^{*}\right)$ is called a generalized quantum stochastic process. A generalized quantum stochastic process $\Xi$ is called regular 
if it admits a continuous extension from $E_{C}^{*}$ into $\mathscr{Z}\left((E),(E)^{*}\right)$. The extension will be denoted by the same symbol.

If $\left\{\Xi_{t} ; t \in \mathbb{R}\right\}$ is a quantum stochastic process, so is $\left\{\Xi_{t}^{*} ; t \in \mathbb{R}\right\}$. This is called the dual process. Similarly, the dual process of a (resp. regular) generalized quantum stochastic process is defined and becomes a (resp. regular) generalized quantum stochastic process.

Lemma 2.2. If a generalized quantum stochastic process $\Xi$ is regular, there exists $p \geq 0$ such that $t \mapsto \Xi_{t}=\Xi\left(\delta_{t}\right) \in \mathscr{L}_{p}^{\prime}\left((E),(E)^{*}\right)$ is continuous. In particular, $\left\{\Xi_{t}\right\}$ is a quantum stochastic process.

Proof. By the canonical isomorphism $\mathscr{L}\left((E),(E)^{*}\right) \cong((E) \otimes(E))^{*}$ we consider a continuous linear map $\Xi^{*}:(E) \otimes(E) \rightarrow E_{C}$. (Here $\Xi^{*}$ does not stand for the dual process.) Hence for any $p \geq 0$ there exist $C \geq 0$ and $q \geq 0$ such that

$$
\left|\Xi^{*}(\omega)\right|_{p} \leq C\|\omega\|_{p+q}, \quad \omega \in(E) \otimes(E) .
$$

Then, by duality we have

$$
\|\Xi(f)\|_{-(p+q)} \leq C|f|_{-p}, \quad f \in E_{C}^{*} .
$$

It is known that $t \mapsto \delta_{t} \in E_{-p}$ is continuous whenever $p>5 / 12$, see Appendix A.2. For such $p$ and $q$ as above, the map $t \mapsto \Xi_{t} \in \mathcal{Z}_{p+q}\left((E),(E)^{*}\right)$ is continuous. qed

Not every quantum stochastic process is of the form of the above lemma. From now on a regular generalized quantum stochastic process is also called a regular quantum stochastic process.

A $C$-valued measurable function $f$ is called slowly increasing if $\lim _{|x| \rightarrow \infty}\left(1+t^{2}\right)^{-\alpha}|f(t)|=0$ for some $\alpha>0$. Every slowly increasing function belongs to $E_{C}^{*}$ and, as usual we write

$$
\langle f, \xi\rangle=\int_{R} f(t) \xi(t) d t, \quad \xi \in E_{C} .
$$

Lemma 2.3. Let $\Xi$ be a regular generalized quantum stochastic process. Then for any slowly increasing function $f$ it holds that

$$
\left\langle\Xi(f) \phi, \psi 》=\int_{R} f(t) 《 \Xi_{t} \phi, \psi 》 d t, \quad \phi, \psi \in(E) .\right.
$$

Proof. As in the proof of Lemma 2.2 let $\Xi^{*}:(E) \otimes(E) \rightarrow E_{C}$ denote the adjoint of $\Xi$. Then

$$
\left\langle\Xi_{t} \phi, \psi\right\rangle=\left\langle\Xi\left(\delta_{t}\right) \phi, \psi 》=\left\langle\left\langle\Xi\left(\delta_{t}\right), \phi \otimes \psi 》=\left\langle\delta_{t}, \Xi^{*}(\phi \otimes \psi)\right\rangle=\Xi^{*}(\phi \otimes \psi)(t),\right.\right.\right.
$$

which belongs to $E_{C}$, i.e., is a rapidly decreasing function of $t$. Therefore 


$$
\begin{aligned}
\left.\int_{R} f(t) 《 \Xi_{t} \phi, \psi\right\rangle d t & =\int_{R} f(t) \Xi^{*}(\phi \otimes \psi)(t) d t=\left\langle f, \Xi^{*}(\phi \otimes \psi)\right\rangle \\
& =\langle\Xi(f), \phi \otimes \psi\rangle=\langle\Xi(f) \phi, \psi\rangle,
\end{aligned}
$$

as desired.

\section{§2.2. Basic Examples}

Example 1. It follows from Lemma A.3 that

$$
f \mapsto \Xi_{0,1}(f)=\int_{R} f(t) \partial_{t} d t \in \mathscr{L}^{\prime}((E),(E)), \quad f \in E_{C}^{*},
$$

is a continuous linear map. Therefore, by definition $\Xi_{0,1}$ is a regular generalized quantum stochastic process. In particular, $\left\{\partial_{t}=\Xi_{0,1}\left(\delta_{t}\right)\right\}$ is a regular quantum stochastic process. In that case $t \mapsto \partial_{1} \in \mathcal{Y}^{\prime}((E),(E))$ is continuous. Similarly $\Xi_{1,0}$ is a regular generalized quantum stochastic process which is the dual process of $\Xi_{0.1}$. In particular, $\left\{\partial_{t}^{*}\right\}$ is the dual process of $\left\{\partial_{t}\right\}$ and $t \mapsto \partial_{t}^{*} \in \mathscr{L}^{\prime}\left((E)^{*},(E)^{*}\right)$ is continuous.

It is known that each $\Phi \in(E)^{*}$ is regarded as a continuous operator in $\mathscr{Z}\left((E),(E)^{*}\right)$ by multiplication. The operator is denoted by the same symbol. Then by definition

$$
\langle\Phi \phi, \psi\rangle=\left\langle\Phi, \phi \psi 》, \quad \phi, \psi \in(E), \quad \Phi \in(E)^{*} .\right.
$$

Thus we obtain a natural injection: $(E)^{*} \rightarrow \downarrow\left((E),(E)^{*}\right)$. It is known that $\Phi \in$ $\mathcal{Y}((E),(E))$ if and only if $\Phi \in(E)$. Furthermore, we have the following commutative diagram:

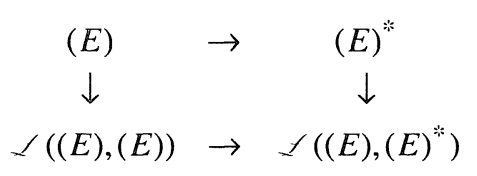

where all arrows are continuous linear maps.

Example 2. Let $t \mapsto \Phi_{1} \in(E)^{*}$ be a continuous flow. Then, regarded as multiplication operators, it forms a quantum stochastic process. This is the standard way to regard a classical (generalized) stochastic process as a quantum stochastic process. In particular, the white noise $\{x(t)\}$ regarded as multiplication operators is a quantum stochastic process. This is called the quantum white noise. The well-known relation:

$$
x(t)=\partial_{t}+\partial_{t}^{*}, \quad t \in \mathbb{R},
$$

implies that the quantum white noise is regular. 
Example 3. Consider the integral kernel operators:

$$
A_{t}=\int_{R} 1_{\{0, t]}(s) \partial_{,} d s=\int_{0}^{t} \partial_{,} d s, \quad A_{t}^{*}=\int_{R} 1_{[0, t]}(s) \partial_{,}^{*} d s=\int_{0}^{t} \partial_{i}^{*} d s,
$$

which are called the annihilation and creation processes, respectively. These processes will play a central role in quantum stochastic integrals. In the work of Hudson-Parthasarathy [12] a crucial role is played also by the number (gauge) process which is expressed as an integral kernel operator:

$$
\Lambda_{t}=\int_{R^{2}} \tau_{[0, t]}(s, u) \partial_{1}^{*} \partial_{u} d s d u=\int_{0}^{t} \partial_{i}^{*} \partial_{,} d s
$$

where $\tau_{[0, t]} \in(E \otimes E)^{*}=\mathcal{P}^{\prime}\left(\boldsymbol{R}^{2}\right)$ is defined by

$$
\left\langle\tau_{[0, t]}, \zeta\right\rangle=\int_{0}^{t} \zeta(s, s) d s, \quad \zeta \in E \otimes E=\mathscr{P}\left(\boldsymbol{R}^{2}\right) .
$$

Obviously, $\Lambda_{t}^{*}=\Lambda_{t}$. It is straightforward to verify that the above $\left\{A_{t}\right\},\left\{A_{t}^{*}\right\}$ and $\left\{\Lambda_{t}\right\}$ constitute quantum stochastic processes. The integral interval $[0, t]$ can be replaced with an arbitrary finite interval $[a, t]$ or $(-\infty, t]$. In $\S 3$ we discuss another aspects of these processes.

Example 4. The quantum stochastic process defined as

$$
Q_{t}=A_{t}+A_{t}^{*}=\int_{0}^{t}\left(\partial_{1}+\partial_{1}^{*}\right) d s, \quad t \geq 0,
$$

is called the quantum Brownian motion or the position process. It applies to the vacuum $\phi_{0} \in(E)$ to obtain a classical Brownian motion. In fact,

$$
Q_{t} \phi_{0}(x)=\left\langle x, 1_{[0, t]}\right\rangle=B_{t}(x), \quad x \in E^{*}, \quad t \geq 0,
$$

whence $\mathbf{E}\left(B_{t}\right)=0$ and $\mathbf{E}\left(B_{,} B_{t}\right)=s \wedge t$ for $s, t \geq 0$. Therefore the quantum Brownian motion $Q_{t}$ coincides with $B_{t}$ as multiplication operator.

Example 5. Let $l \geq 0$ be a constant. The quantum stochastic process defined as

$$
P_{t}=\Lambda_{t}+\sqrt{l} Q_{t}+l t=\int_{0}^{t}\left(\partial_{1}^{*} \partial_{1}+\sqrt{l}\left(\partial_{1}^{*}+\partial_{1}\right)+l\right) d s,
$$

is called the quantum Poisson process. For the naming see [12], [26].

\section{§2.3. Fock Expansion of Quantum Stochastic Processes}

The theory of Fock expansion [23] says that every $\Xi \in \mathscr{L}^{\prime}\left((E),(E)^{*}\right)$ admits an expansion in terms of integral kernel operators: 


$$
\Xi=\sum_{l, m=0}^{\infty} \Xi_{l, m}\left(\kappa_{l, m}\right)
$$

where the series converges in $\mathcal{Z}\left((E),(E)^{*}\right)$ and the kernel distributions $\kappa_{l, m} \in$ $\left(E_{C}^{\otimes(I+m)}\right)_{\mathrm{hym}(l, m)}^{*}$ are uniquely determined. For $\Xi \in \mathcal{Z}((E),(E))$ the kernel distribution $\kappa_{l, m}$ belongs to $\left(E_{C}^{\otimes l}\right) \otimes\left(E_{C}^{\otimes m}\right)^{*}$, namely, $\Xi_{l, m}\left(\kappa_{l, m}\right) \in \mathcal{Z}((E),(E))$ by Theorem 1.6 and the series converges in $\mathcal{Z}((E),(E))$. For $\Xi \in \mathcal{Z}\left((E),(E)^{*}\right)$ the function on $E_{C} \times E_{C}$ defined by

$$
\hat{\Xi}(\xi, \eta)=《 \Xi \phi_{\xi}, \phi_{\eta} 》, \quad \xi, \eta \in E_{C},
$$

is called the symbol of $\Xi$. Since the exponential vectors $\left\{\phi_{\xi} ; \xi \in E_{C}\right\}$ span a dense subspace of $(E)$, the symbol recovers the operator uniquely. If $\Xi \in \mathscr{L}_{p}((E)$, $\left.(E)^{*}\right), p \geq 0$, we have

$$
|\hat{\Xi}(\xi, \eta)| \leq\|\Xi\|_{-p} \exp \frac{1}{2}\left(|\xi|_{p}^{2}+|\eta|_{p}^{2}\right), \quad \xi, \eta \in E_{C} .
$$

In fact,

$$
|\hat{\Xi}(\xi, \eta)|=\left|\left\langle\Xi \phi_{\xi}, \phi_{\eta}\right\rangle\right|=\left|\left\langle\Xi, \phi_{\xi} \otimes \phi_{\eta}\right\rangle\right| \leq\|\Xi\|_{-p}\left\|\phi_{\xi} \otimes \phi_{\eta}\right\|_{p},
$$

from which (2.7) follows immediately. Then, applying the result in [22, §5] or [23, Theorem 4.4.6] with (2.7), we come to the following

Lemma 2.4. For $\Xi \in \mathcal{I}_{p}\left((E),(E)^{*}\right), p \geq 0$, let $\Xi=\sum_{l, m=0}^{\infty} \Xi_{l, m}\left(\kappa_{l, m}\right)$ be the Fock expansion. Then

$$
\left|\kappa_{l, m}\right|_{-(p+1)} \leq G_{l, m, p}\|\Xi\|_{-p},
$$

where

$$
G_{l, m, p}=\left(l^{l} m^{m}\right)^{-1 / 2}\left(e^{3} \delta^{2}\left(1+\rho^{2 p}\right)\right)^{(l+m) / 2} .
$$

We now consider the Fock expansion of a generalized quantum stochastic process.

Proposition 2.5. Let $\Xi$ be a generalized quantum stochastic process and let

$$
\Xi(\xi)=\sum_{l, m=0}^{\infty} \Xi_{l, m}\left(\kappa_{l, m}(\xi)\right), \quad \xi \in E_{C}
$$

be the Fock expansion. Then, there exist $C \geq 0$ and $p \geq 0$ such that

$$
\left|\kappa_{l, m}(\xi)\right|_{-(p+1)} \leq C G_{l, m, p}|\xi|_{p}, \quad \xi \in E_{C}
$$


where $G_{l, m, p}$ is defined in (2.9). In particular, $\xi \mapsto \kappa_{l, m}(\xi)$ is a continuous linear map, namely, $\kappa_{l, m} \in \mathscr{L}^{\prime}\left(E_{C},\left(E_{C}^{\otimes(l+m)}\right)_{\text {sym }(l, m)}^{*}\right)$.

Proof. By definition $\Xi: E_{C} \rightarrow \mathscr{L}^{\prime}\left((E),(E)^{*}\right) \cong((E) \otimes(E))^{*}$ is continuous. Hence by the general theory of countable Hilbert spaces (see e.g., [6, Chapter I]), there exist $p \geq 0$ and $C \geq 0$ such that

$$
\|\Xi(\xi)\|_{-p} \leq C|\xi|_{p}, \quad \xi \in E_{C} .
$$

In that case $\Xi(\xi) \in \mathscr{L}_{p}((E),(E))$ for any $\xi \in E_{C}$. Then (2.11) follows from Lemma 2.4 and (2.12).

qed

Suppose that a generalized quantum stochastic process $\Xi$ is regular. Then, for any $p \geq 0$ there exist $q \geq 0$ and $C \geq 0$ such that

$$
\|\Xi(f)\|_{-(p+q)} \leq C|f|_{-p}, \quad f \in E_{C}^{*},
$$

see the proof of Lemma 2.2. Then, modifying the proof of Proposition 2.5, one obtains the following

$\mathbb{P r} \propto p o s i t i o n$ 2.6. Let $\Xi$ be a regular generalized quantum stochastic process with Fock expansion given as in (2.10). Then for any $p \geq 0$ there exist $q \geq 0$ and $C \geq 0$ such that

$$
\left|\kappa_{l, m}(f)\right|_{-(p+q+1)} \leq C G_{l, m, p+q}|f|_{-p}, \quad f \in E_{C}^{*} .
$$

In particular, the map $\xi \mapsto \kappa_{l, m}(\xi)$ is continuously extended to a linear map from $E_{C}^{*}$ into $\left(E_{C}^{\otimes(l+m)}\right)_{\mathrm{hym}(l, m)}^{*}$, namely, $\kappa_{l, m} \in \mathcal{Z}\left(E_{C}^{*},\left(E_{C}^{\otimes(l+m)}\right)_{\mathrm{hym}(l, m)}^{*}\right)$.

As for a quantum stochastic process we only mention the following

Propositiom 2.7. Let $\left\{\Xi_{t}\right\}$ be a quantum stochastic process and let

$$
\Xi_{t}=\sum_{l, m=0}^{\infty} \Xi_{l, m}\left(\kappa_{l, m}(t)\right)
$$

be the Fock expansion. Assume that for any finite interval $[a, b]$ there exists $p \geq 0$ such that $t \mapsto \Xi_{t} \in \mathcal{L}_{p}\left((E),(E)^{*}\right)$ is continuous on $[a, b]$. Then $t \mapsto \kappa_{l, m}(t) \in$ $\left(E_{C}^{\otimes(l+m)}\right)_{\mathrm{sym}(l, m)}^{*}$ is continuous on $[a, b]$.

Proof. Since

$$
\Xi_{1}-\Xi_{t}=\sum_{l, m=0}^{\infty} \Xi_{l, m}\left(\kappa_{l, m}(s)-\kappa_{l, m}(t)\right)
$$

is the Fock expansion of $\Xi_{1}-\Xi_{t} \in \mathscr{L}_{p}^{\prime}\left((E),(E)^{*}\right)$, we see from Lemma 2.4 that

$$
\left|\kappa_{l, m}(s)-\kappa_{l, m}(t)\right|_{-(p+1)} \leq G_{l, m, p}\left\|\Xi_{,}-\Xi_{t}\right\|_{-p}, \quad s, t \in[a, b] .
$$


Then the assertion is obvious.

qed

\section{§2.4. Stochastic Integral-like Representation}

For a generalized quantum stochastic process $\Xi$ we consider the Fock expansion divided into three parts:

$$
\Xi(\xi)=\sum_{l \geq 0, m \geq 1} \Xi_{l, m}\left(\kappa_{l, m}(\xi)\right)+\sum_{l \geq 1} \Xi_{l, 0}\left(\kappa_{l, 0}(\xi)\right)+\Xi_{0,0}\left(\kappa_{0,0}(\xi)\right), \quad \xi \in E_{C} .
$$

Note that the third term is a scalar operator. Since

$$
\xi \mapsto\left\langle\left\langle\Xi_{0.0}\left(\kappa_{0.0}(\xi)\right) \phi_{0}, \phi_{0}\right\rangle=\left\langle\left\langle\Xi(\xi) \phi_{0}, \phi_{0}\right\rangle\right.\right.
$$

is a continuous linear functional on $E_{C}$, there exists $c \in E_{C}^{*}$ such that

$$
\Xi_{0,0}\left(\kappa_{0,0}(\xi)\right)=\langle c, \xi\rangle I, \quad \xi \in E_{C} .
$$

The first and second terms in (2.13) are respectively rewritten as generalized integral kernel operators. The main purpose of this section is to prove the following

Theorem 2.8. Let $\Xi$ be a generalized quantum stochastic process. Then there exist $L \in \mathscr{B}\left(E_{C}, E_{C} ; \mathscr{Z}\left((E),(E)^{*}\right)\right), \quad M \in \mathscr{B}\left(E_{C}, E_{C} ; \mathscr{Y}((E),(E))\right)$ with $\left[M(\xi, \eta), \partial_{1}\right]=0$ for all $\xi, \eta \in E_{C}$ and $t \in \mathbb{R}$, and $c \in E_{C}^{*}$ such that

$$
\Xi(\xi)=\int_{R} L(\xi, t) \partial_{t} d t+\int_{R} \partial_{,}^{*} M^{*}(\xi, s) d s+\langle c, \xi\rangle I, \quad \xi \in E_{C} .
$$

(The integrals are generalized integral kernel operators for a fixed $\xi \in E_{C}$, see $\S 1.3)$

The proof is divided into Lemmas 2.9-2.11 below.

Lemma 2.9. There exists $L \in \mathscr{B}\left(E_{C}, E_{C} ; \mathscr{Z}\left((E),(E)^{*}\right)\right)$ such that

$$
\int_{R} L(\xi, t) \partial_{t} d t=\sum_{l \geq 0, m \geq 1} \Xi_{l, m}\left(\kappa_{l, m}(\xi)\right), \quad \xi \in E_{C} .
$$

Proof. By Proposition 2.5 there exist $C \geq 0$ and $p \geq 0$ such that

$$
\left|\kappa_{l, m}(\xi)\right|_{-(p+1)} \leq C G_{l, m, p}|\xi|_{p}, \quad \xi \in E_{C} .
$$

We fix such a pair of $C \geq 0$ and $p \geq 0$ throughout. For $l \geq 0, m \geq 1$ we put

$$
L_{l, m}(\xi, \eta)=\Xi_{l, m-1}\left(\kappa_{l, m}(\xi) \otimes_{1} \eta\right), \quad \xi \in E_{C} .
$$

Letting $\xi \in E_{\boldsymbol{C}}$ be fixed, we apply the Fubini type theorem (Theorem 1.8) to obtain 


$$
\Xi_{l, m}\left(\kappa_{l, m}(\xi)\right)=\int_{R} L_{l, m}(\xi, t) \partial_{t} d t
$$

We shall prove that the infinite series

$$
\sum_{l \geq 0, m \geq 1} L_{l, m}(\xi, \eta) \phi, \quad \xi, \eta \in E_{C}, \quad \phi \in(E)
$$

converges in $(E)^{*}$. For $q \geq 0$ we see from Lemma A.2 that

$$
\begin{aligned}
\left\|L_{l, m}(\xi, \eta) \phi\right\|_{-(p+q+1)} & =\left\|\Xi_{l, m-1}\left(\kappa_{l, m}(\xi) \otimes_{1} \eta\right) \phi\right\|_{-(p+q+1)} \\
& \leq C_{l, m-1, p+q+1}\left|\kappa_{l, m}(\xi) \otimes_{1} \eta\right|_{-(p+q+1)}\|\phi\|_{p+q+1} .
\end{aligned}
$$

On the other hand, by (1.16) we have

$$
\left|\kappa_{l, m}(\xi) \otimes_{1} \eta\right|_{-(p+q+1)} \leq\left|\kappa_{l, m}(\xi)\right|_{-(p+q+1)}|\eta|_{p+q+1},
$$

and by the definition of the norms we have

$$
\left|\kappa_{l, m}(\xi)\right|_{-(p+q+1)} \leq \rho^{(l+m) q}\left|\kappa_{l, m}(\xi)\right|_{-(p+1)} .
$$

Then, inserting (2.16), (2.21) and (2.22) into (2.20), we obtain

$$
\left\|L_{l, m}(\xi, \eta) \phi\right\|_{-(p+q+1)} \leq \rho^{(l+m) q} C_{l, m-1 ; p+q+1} C G_{l, m, p}|\xi|_{p}|\eta|_{p+q+1}\|\phi\|_{p+q+1} .
$$

Using the explicit forms of $C_{l, m-1 . p+q+1}$ and $G_{l, m, p}$ (see (2.9) and Appendix A.1), we have

$$
\rho^{(l+m) q} C_{l, m-1, p+q+1} G_{l, m, p} \leq\left(\frac{\rho^{p+q+1}}{-2(p+q+1) e \log \rho}\right)^{-1 / 2}\left(\frac{e^{3} \delta^{2}\left(1+\rho^{2 p}\right) \rho^{-(p+1)} \rho^{q}}{-2(p+q+1) e \log \rho}\right)^{(1+m) / 2}
$$

Take a sufficiently large $q \geq 0$ such that

$$
\frac{e^{3} \delta^{2}\left(\rho^{2 p}+1\right) \rho^{-(p+1)} \rho^{q}}{-2(p+q+1) e \log \rho}<1
$$

Then, summing up both sides of (2.23) we come to

$$
\sum_{l \geq 0, m \geq 1}\left\|L_{l, m}(\xi, \eta) \phi\right\|_{-(p+q+1)} \leq C^{\prime}|\xi|_{p}|\eta|_{p+q+1}\|\phi\|_{p+q+1}, \quad \xi, \eta \in E_{C}, \quad \phi \in(E),
$$

where

$$
C^{\prime}=C\left(\frac{\rho^{p+q+1}}{-2(p+q+1) e \log \rho}\right)^{-1 / 2} \sum_{\mid \geq 0, m \geq 1}\left(\frac{e^{3} \delta^{2}\left(1+\rho^{2 p}\right) \rho^{-(p+1)} \rho^{q}}{-2(p+q+1) e \log \rho}\right)^{(1+m) / 2}<\infty .
$$

We have thus proved that the infinite series (2.19) converges in $(E)^{*}$.

We now put

$$
B(\xi, \eta, \phi)=\sum_{l \geq 0, m \geq 1} L_{l, m}(\xi, \eta) \phi, \quad \xi, \eta \in E_{C}, \phi \in(E) .
$$

Then it follows from (2.24) that 


$$
\|B(\xi, \eta, \phi)\|_{-(p+q+1)} \leq C^{\prime}|\xi|_{p}|\eta|_{p+q+1}\|\phi\|_{p+q+1}, \quad \xi, \eta \in E_{C}, \phi \in(E) .
$$

In other words, the 3-linear map $B: E_{C} \times E_{C} \times(E) \rightarrow(E)^{*}$ is continuous. By the repeated application of the kernel theorem, there exists a unique $L \in \mathscr{乃}\left(E_{C}, E_{C}\right.$; $\left.\mathscr{L}\left((E),(E)^{*}\right)\right)$ such that

$$
L(\xi, \eta) \phi=B(\xi, \eta, \phi)=\sum_{l \geq 0, m \geq 1} L_{l, m}(\xi, \eta) \phi
$$

This $L$ is what we are seeking. In fact, by definition (1.14) we have

$$
\left.《\left(\int_{R} L(\xi, t) \partial_{t} d t\right) \phi_{\eta}, \phi_{\zeta} 》\right\rangle=\ll L(\xi, \eta) \phi_{\eta}, \phi_{\zeta} 》, \quad \eta, \zeta \in E_{C} .
$$

On the other hand, a straightforward computation with (2.25) and (2.17) yields

$$
\left\langle L(\xi, \eta) \phi_{\eta}, \phi_{\zeta}\right\rangle=\sum_{l \geq 0, m \geq 1}\left\langle\left\langle\Xi_{l, m}\left(\kappa_{l, m}(\xi)\right) \phi_{\eta}, \phi_{\zeta} 》 .\right.\right.
$$

Since $\sum_{l \geq 0, m \geq 1} \Xi_{l, m}\left(\kappa_{l, m}(\xi)\right) \phi$ converges in $(E)^{*}$ for any $\xi \in E_{C}$ and $\phi \in(E)$, the last expression coincides with the symbol of $\sum_{l \geq 0, m \geq 1} \Xi_{l, m}\left(\kappa_{l, m}(\xi)\right)$, and therefore identity (2.15) holds.

qed

Lemma 2.10. There exists $M \in \mathscr{B}\left(E_{C}, E_{C} ; \mathcal{Z}((E),(E))\right)$ such that

$$
M(\xi, \eta) \phi=\sum_{l \geq 1} \Xi_{0 . l-1}\left(\kappa_{l, 0}(\xi) \otimes_{1} \eta\right) \phi, \quad \phi \in(E)
$$

where the right hand side converges in $(E)$. Moreover, $\left[M(\xi, \eta), \partial_{t}\right]=0$ for any $\xi, \eta \in E_{C}$ and $t \in \boldsymbol{R}$.

Proof. Let $C \geq 0$ and $p \geq 0$ be the same as in the proof of Lemma 2.9. For simplicity we put

$$
M_{l}(\xi, \eta)=\Xi_{0, l-1}\left(\kappa_{l, 0}(\xi) \otimes_{1} \eta\right), \quad \xi, \eta \in E_{C}, l \geq 1 .
$$

We follow the proof of Lemma 2.9 using Lemma A.3 instead of Lemma A.2 to obtain

$$
\left\|M_{l}(\xi, \eta) \phi\right\|_{p+1} \leq C_{l-1, q}\left|\kappa_{l, 0}(\xi)\right|_{-(p+q+1)}|\eta|_{p+q+1}\|\phi\|_{p+q+1}
$$

for any $q>0, r \geq 0, \xi \in E_{C}$ and $\phi \in(E)$. Replacing $q$ with $q+1, q \geq 0$, and in view of (2.16) we obtain

$$
\begin{aligned}
\left\|M_{l}(\xi, \eta) \phi\right\|_{p+1} & \leq \rho^{((q+1)} C_{l-1, q+1}\left|\kappa_{l, 0}(\xi)\right|_{-(p+1)}|\eta|_{p+q+1+1}\|\phi\|_{p+q+1+1} \\
& \leq \rho^{l(q+1)} C_{l-1, q+1} C G_{l, 0, p}|\xi|_{p}|\eta|_{p+q+++1}\|\phi\|_{p+q+1+1}
\end{aligned}
$$

On the other hand, by explicit computation we have

$$
\rho^{l q} C_{l-1, q+1} G_{I, 0, p} \leq(-2(q+1) e \log \rho)^{1 / 2}\left(\frac{\rho^{q+2,-1} e^{3} \delta^{2}\left(1+\rho^{2 p}\right)}{-2(q+1) e \log \rho}\right)^{1 / 2} .
$$


Take a sufficiently large $q \geq 0$ such that

$$
\frac{\rho^{q+2,-1} e^{3} \delta^{2}\left(1+\rho^{2 p}\right)}{-2(q+1) e \log \rho}<1
$$

Then, summing up both sides of (2.27), we obtain

$$
\sum_{l \geq 1}\left\|M_{l}(\xi, \eta) \phi\right\|_{p+l} \leq C^{\prime \prime}|\xi|_{p}|\eta|_{p+q+1+1}\|\phi\|_{p+q+1+1}, \quad \xi, \eta \in E_{C}, \quad \phi \in(E),
$$

where

$$
C^{\prime \prime}=C(-2(q+1) e \log \rho)^{1 / 2} \sum_{l \geq 1}\left(\frac{\rho^{q+2,-1} e^{3} \delta^{2}\left(1+\rho^{2 p}\right)}{-2(q+1) e \log \rho}\right)^{1 / 2}<\infty
$$

Therefore

$$
B(\xi, \eta, \phi)=\sum_{l \geq 1} M_{l}(\xi, \eta) \phi, \quad \xi, \eta \in E_{C}, \quad \phi \in(E),
$$

converges in $(E)$ and $B$ becomes a continuous 3-linear map from $E_{C} \times E_{C} \times(E)$ into $(E)$. Then, in a similar manner as in the proof of Lemma 2.9 we see that there exists $M \in \mathscr{B}\left(E_{C}, E_{C} ; \mathscr{Z}((E),(E))\right)$ such that $B(\xi, \eta, \phi)=M(\xi, \eta) \phi$, namely, (2.26) holds. Since the integral kernel operator $M_{l}(\xi, \eta)$ contains only annihilation operators by construction, $\left[M_{l}(\xi, \eta), \partial_{t}\right]=0$ and therefore $[M(\xi, \eta)$, $\left.\partial_{t}\right]=0$.

For $M \in \mathscr{B}\left(E_{C}, E_{C} ; \mathscr{L}((E),(E))\right)$ define $M^{*} \in \mathscr{B}\left(E_{C}, E_{C} ; \mathscr{L}\left((E)^{*},(E)^{*}\right)\right)$ by

$$
\left\langle M^{*}(\xi, \eta) \Phi, \phi 》=\left\langle\Phi, M(\xi, \eta) \phi 》, \quad \xi, \eta \in E_{C}, \quad \phi \in(E), \quad \Phi \in(E)^{*} .\right.\right.
$$

Lemma 2.11. It holds that

$$
\int_{R} \partial_{1}^{*} M^{*}(\xi, s) d s=\sum_{l \geq 1} \Xi_{l, 0}\left(\kappa_{l, 0}(\xi)\right), \quad \xi \in E_{C}
$$

The proof is to show the coincidence of the symbols by a straightforward computation, cf. the proof of Lemma 2.9. We have thus completed the proof of Theorem 2.8.

By similar arguments we have the following results.

Theorem 2.12. Let $\Xi$ be a regular generalized quantum stochastic process. Then there exist $L \in \mathscr{H}_{\text {sep }}\left(E_{C}^{*}, E_{C} ; \mathscr{Z}^{\prime}\left((E),(E)^{*}\right)\right), \quad M \in \mathscr{B}_{\text {sep }}\left(E_{C}^{*}, E_{C} ; \mathscr{Z}^{\prime}((E)\right.$, $(E)))$ with $\left[M(f, \eta), \partial_{t}\right]=0$ for all $f \in E_{C}^{*}, \eta \in E_{C}, t \in \mathbb{R}$ and $c \in E_{C}$ such that (2.14) holds. In particular,

$$
\Xi_{t}=\int_{R} L(t, s) \partial_{1} d s+\int_{R} \partial^{*} M^{*}(t, s) d s+c_{t} I, \quad t \in \mathbb{R}
$$

where $c_{t}=\left\langle\delta_{t}, c\right\rangle=\left\langle\Xi_{t} \phi_{0}, \phi_{0}\right\rangle$. 
Theorem 2.13. Let $\left\{\Xi_{t}\right\}$ be a quantum stochastic process. Assume that for any finite interval $[a, b]$ there exists $p \geq 0$ such that $t \mapsto \Xi_{t} \in \mathscr{Z}_{p}\left((E),(E)^{*}\right)$ is continuous on $[a, b]$. Then there exist continuous maps $t \mapsto L_{t} \in \mathscr{L}^{\prime}\left(E_{C}, \mathscr{L}^{\prime}((E)\right.$, $\left.\left.(E)^{*}\right)\right), t \mapsto M_{t} \in \mathscr{Z}^{\prime}\left(E_{C}, \mathscr{L}^{\prime}((E),(E))\right)$ and $t \mapsto c_{t} \in C, t \in[a, b]$, such that

$$
\Xi_{t}=\int_{R} L_{t}(s) \partial_{,} d s+\int_{R} \partial_{i}^{*} M_{t}^{*}(s) d s+c_{t} I, \quad t \in[a, b] .
$$

\section{§3. Quantum Stochastic Integrals}

\section{§3.1. Integrals against Time}

In this section we discuss integration of a quantum stochastic process $\left\{L_{\mathrm{s}}\right\}$ against $d s$. Let $L_{\text {loc }}^{1}(\mathbb{R})$ be the space of all $\boldsymbol{C}$-valued locally integrable functions on $\boldsymbol{R}$. We begin with the following

Lemma 3.1. Let $\left\{L_{t}\right\}$ be a quantum stochastic process. Then for any $a, b \in \mathbb{R}$ and $f \in L_{\mathrm{loc}}^{1}(\mathbb{R})$ there exists a unique operator $\Xi_{a, b}(f) \in \mathcal{Z}\left((E),(E)^{*}\right)$ such that

$$
\left.\left\langle\Xi_{a, b}(f) \phi, \psi\right\rangle=\int_{a}^{b} f(s) 《 L, \phi, \psi\right\rangle d s, \quad \phi, \psi \in(E) .
$$

Proof. Suppose $a, b$ are fixed. Since $s \mapsto L$ is continuous, the closed interval $[a, b]$ is mapped to a compact subset $K \subset \mathcal{Y}\left((E),(E)^{*}\right) \cong((E) \otimes(E))^{*}$. Hence there exists some $p \geq 0$ such that

$$
C \equiv \sup _{a \leq i \leq b}\left\|L_{\triangleleft}\right\|_{-p}<\infty .
$$

Then for any $s \in[a, b]$ we have

$$
||\left\langle L_{\imath} \phi, \psi\right\rangle|=|\left\langle L_{1}, \phi \otimes \psi\right\rangle \mid \leq\left\|L_{1}\right\|_{-p}\|\phi \otimes \psi\|_{p} \leq C\|\phi\|_{p}\|\psi\|_{p},
$$

and

$$
\left|\int_{a}^{b} f(s)\left\langle L_{1} \phi, \psi\right\rangle d s\right| \leq C\|\phi\|_{p}\|\psi\|_{p} \int_{a}^{b}|f(s)| d s, \quad \phi, \psi \in(E) .
$$

Therefore the right hand side of (3.1) is a continuous bilinear form on $(E)$ and there exists a unique operator $\Xi_{a, b}(f) \in \mathcal{Y}\left((E),(E)^{*}\right)$ such that $(3.1)$ holds. qed

The above constructed operator $\Xi_{a, b}(f)$ is denoted as

$$
\Xi_{a, b}(f)=\int_{a}^{b} f(s) L, d s \text {. }
$$


Unless the continuity of $t \mapsto f(t) \Xi_{t} \in \mathcal{Z}^{\prime}\left((E),(E)^{*}\right)$ is assumed, $\{f(t) \Xi(t)\}$ does not necessarily constitute a quantum stochastic process in our sense. The next result follows immediately from the definition.

Lemma 3.2. Let $\left\{L_{t}\right\}$ be a generalized quantum stochastic process. Then

$$
\left(\int_{a}^{b} f(s) L_{\mathrm{s}} d s\right)^{*}=\int_{a}^{b} f(s) L_{\mathrm{s}}^{*} d s
$$

for any $f \in L_{\mathrm{loc}}^{1}(\boldsymbol{R})$ and $a \leq b$.

Theorem 3.3. Let $\left\{L_{t}\right\}$ be a quantum stochastic process and let $f \in L_{\mathrm{loc}}^{1}(\boldsymbol{R})$. Then for any fixed $t_{0} \in \boldsymbol{R}$,

$$
\Xi_{t}=\int_{t_{0}}^{t} f(s) L, d s, \quad t \in \boldsymbol{R},
$$

is a quantum stochastic process.

Proof. It is sufficient to prove the continuity on any finite interval $(a, b)$. Taking constant numbers $p \geq 0, C \geq 0$ as in the proof of Lemma 3.1, we obtain

$$
\left|\left\langle\left(\Xi_{t_{2}}-\Xi_{t_{1}}\right) \phi, \psi\right\rangle\right| \leq C\|\phi\|_{p}\|\psi\|_{p} \int_{t_{1}}^{t_{2}}|f(s)| d s, \quad a<t_{1} \leq t_{2}<b, \quad \phi, \psi \in(E) .
$$

Then for bounded subsets $B_{1}, B_{2} \subset(E)$ we have

$$
\left\|\Xi_{t_{1}}-\Xi_{t_{2}}\right\|_{B_{1}, B_{2}} \leq C\left\|B_{1}\right\|_{p}\left\|B_{2}\right\|_{p} \int_{t_{1}}^{t_{2}}|f(s)| d s, \quad a<t_{1} \leq t_{2}<b
$$

from which the continuity follows immediately.

qed

Corollary 3.4. Let $L$ be a regular generalized quantum stochastic process. Then

$$
L\left(f \cdot 1_{[a, b]}\right)=\int_{a}^{b} f(s) L, d s, \quad f \in L_{\mathrm{loc}}^{1}(\boldsymbol{R}), \quad a \leq b .
$$

Proof. Modelled after the argument as in the proof of Lemma 2.3.

qed

Remark. Unless a generalized quantum stochastic process $L$ is assumed to be regular, $s \mapsto 1_{[a . t]}(s) L$, is not well defined even as an element in $\mathscr{L}\left(E_{C}, \mathcal{L}((E)\right.$, $\left.\left.(E)^{*}\right)\right)$. In that case we have no way to define

$$
\Xi_{t}=\int_{a}^{t} L_{\imath} d s=\int_{R} 1_{[a, t]}(s) L_{\imath} d s
$$

within the present formulation. 
Theorem 3.5. Assume that two quantum stochastic processes $\left\{L_{t}\right\},\left\{\Xi_{t}\right\}$ are related as

$$
\Xi_{t}=\int_{a}^{t} L_{s} d s, \quad t \in \boldsymbol{R}
$$

Then, the map $t \mapsto \Xi_{t}$ is differentiable with respect to the topology of $\mathscr{L}((E)$, $\left.(E)^{*}\right)$ and it holds that

$$
\frac{d}{d t} \Xi_{t}=L_{t}
$$

Proof. We prove the differentiability at a fixed $t \in \boldsymbol{R}$. For that purpose, it is sufficient to show that given bounded subsets $B_{1}, B_{2} \in(E)$

$$
\lim _{h \rightarrow 0}\left\|\frac{\Xi_{t+h}-\Xi_{t}}{h}-L_{t}\right\|_{B_{1}, B_{2}}=0 \text {. }
$$

It follows from definition that

$$
\left.《\left(\frac{\Xi_{t+h}-\Xi_{t}}{h}-L_{t}\right) \phi, \psi 》\right\rangle=\frac{1}{h} \int_{t}^{t+h} 《\left(L_{s}-L_{t}\right) \phi, \psi 》 d s, \quad \phi, \psi \in(E) .
$$

Since $s \mapsto L$, is continuous, given $\varepsilon>0$ there exists some $\delta>0$ such that

$$
\left\|L_{1}-L_{t}\right\|_{B_{1}, B_{2}}<\varepsilon, \quad|s-t|<\delta .
$$

Hence, if $0<|h|<\delta$, we have

$$
\left\|\frac{\Xi_{t+h}-\Xi_{t}}{h}-L_{t}\right\|_{B_{1}, B_{2}} \leq \frac{1}{h} \int_{t}^{t+h}\left\|L_{1}-L_{t}\right\|_{B_{1}, B_{2}} d s<\varepsilon .
$$

This proves (3.3).

qed

Corollary 3.6. For the annihilation process $\left\{A_{t}\right\}$, the creation process $\left\{A_{t}^{*}\right\}$ and the number process $\left\{\Lambda_{t}\right\}$ defined in (2.2) and (2.3) it holds that

$$
\frac{d}{d t} A_{t}=\partial_{t}, \quad \frac{d}{d t} A_{t}^{*}=\partial_{t}^{*}, \quad \frac{d}{d t} \Lambda_{t}=\partial_{t}^{*} \partial_{t},
$$

with respect to the topology of $\mathscr{Z}\left((E),(E)^{*}\right)$.

\section{§3.2. Quantum Stochastic Integrals}

We begin with the following

Lemma 3.7. If $\left\{L_{t}\right\}$ is a quantum stochastic process, so are both $\left\{L_{t} \partial_{t}\right\}$ and $\left\{\partial_{t}^{*} L_{t}\right\}$. 
Proof. We first prove that $t \mapsto L_{t} \partial_{t} \in \mathcal{Z}\left((E),(E)^{*}\right)$ is continuous. To this end fix $t \in \mathbb{R}$ and finite interval $(a, b)$ containing $t$. Take $p \geq 0$ such that

$$
C \equiv \sup _{a \leq \leq \leq b}\left\|L_{\triangleleft}\right\|_{-p}<\infty,
$$

see the proof of Lemma 3.1. Let $B_{1}, B_{2} \subset(E)$ be bounded subsets. Then, in view of Lemma 1.2 we have

$$
\begin{aligned}
\left\|L_{1} \partial_{1}-L_{t} \partial_{t}\right\|_{B_{1}, B_{2}} & \leq\left\|L_{1}\left(\partial_{1}-\partial_{t}\right)\right\|_{B_{1}, B_{2}}+\left\|\left(L_{1}-L_{t}\right) \partial_{t}\right\|_{B_{1}, B_{2}} \\
& \leq\left\|L_{1}\right\|_{-p}\left\|\partial_{1}-\partial_{t}\right\|_{B_{1}, p}\left\|B_{2}\right\|_{p}+\left\|L_{1}-L_{t}\right\|_{\partial_{t} B_{1}, B_{2}} \\
& \leq C\left\|\partial_{1}-\partial_{t}\right\|_{B_{1}, p}\left\|B_{2}\right\|_{p}+\left\|L_{1}-L_{t}\right\|_{\partial_{1} B_{1}, B_{2}},
\end{aligned}
$$

and therefore

$$
\lim _{s \rightarrow t}\left\|L_{1} \partial_{1}-L_{t} \partial_{t}\right\|_{B_{1} \cdot B_{2}}=0
$$

as desired. That $t \mapsto \partial_{t}^{*} L_{t}$ is continuous follows by duality.

qed

The above lemma and Theorem 3.3 lead us to the following

Definition 3.8. Let $\left\{L_{t}\right\}$ be a quantum stochastic process. Then the quantum stochastic processes defined as

$$
\int_{a}^{t} L_{s} \partial_{1} d s, \quad \int_{a}^{t} \partial_{1}^{*} L_{1} d s
$$

are called the quantum stochastic integrals of $\left\{L_{t}\right\}$ against the annihilation and creation processes, respectively.

Obviously by Lemma 3.2 ,

$$
\left(\int_{a}^{t} L_{i} \partial_{,} d s\right)^{*}=\int_{a}^{t} \partial_{s}^{*} L_{i}^{*} d s
$$

The number process $\left\{\Lambda_{t}\right\}$ defined in (2.3) is the quantum stochastic integral of $\left\{\partial_{t}^{*}\right\}$ against the annihilation process as well as the quantum stochastic integral of $\left\{\partial_{t}\right\}$ against the creation process.

In [16] and [17] Lindsay introduced a quantum Hitsuda-Skorokhod integral by means of the gradient operator $\nabla$. In our context the gradient operator is given as

$$
\nabla \phi(x, t)=\partial_{t} \phi(x), \quad \phi \in(E), \quad x \in E^{*}, \quad t \in \mathbb{R},
$$

and is a continuous operator from $(E)$ into $(E) \otimes E_{C}$, see $[23, \S 5.1]$. It is then easy to see that a quantum Hitsuda-Skorokhod integral introduced by Lindsay coincides with a quantum stochastic integral against the creation process in disregard of technical details. 
In [25] we also introduced the notion of a quantum Hitsuda-Skorokhod integral $\Omega_{t}$ for a regular quantum stochastic process $\left\{L_{t}\right\}$. In fact, $\Omega_{t}$ is defined as a unique operator satisfying

$$
《 \Omega_{t} \phi_{\xi}, \phi_{\eta} 》=《 L\left(1_{[a, t]} \eta\right) \phi_{\xi}, \phi_{\eta} 》, \quad \xi, \eta \in E_{C},
$$

with the help of the characterization theorem of operator symbols. We see easily that this $\Omega_{t}$ is a particular case of a quantum stochastic integral against the creation process:

$$
\Omega_{t}=\int_{a}^{t} \partial_{s}^{*} L_{\mathrm{s}} d s
$$

On the other hand, if $\left\{L_{t}\right\}$ is a regular quantum stochastic process, one may define a quantum Hitsuda-Skorokhod integral over an infinite interval like $(-\infty, t)$ by (3.5).

\section{§3.3. Riemannian Approximation}

For a quantum stochastic process $\left\{L_{t}\right\}$ we shall define a quantum stochastic integral against the annihilation process $\left\{A_{t}\right\}$ by Riemannian approximation. Let $[a, b]$ be a fixed interval. For a partition

$$
\Delta: a=s_{0}<s_{1}<\cdots<s_{n}=b,
$$

we consider the Riemannian sum of Itô type:

$$
\Xi_{\Delta}=\sum_{j=0}^{n-1} L_{s,}\left(A_{s_{l+1}}-A_{s,}\right)=\sum_{j=0}^{n-1} L_{s} \int_{j,}^{s_{1+1}} \partial_{u} d u
$$

Obviously, $\Xi_{\Delta} \in \mathscr{L}^{\prime}\left((E),(E)^{* *}\right)$. The symbol is given as

$$
\widehat{\Xi_{\Delta}}(\xi, \eta)=\left\langle\left\langle\Xi_{\Delta} \phi_{\xi}, \phi_{\eta}\right\rangle\right\rangle=\sum_{l=0}^{n-1} \widehat{L_{1},}(\xi, \eta) \int_{1,}^{1_{1+1}} \xi(u) d u .
$$

Lemma 3.9. Let $\left\{L_{t}\right\}$ be a quantum stochastic process. Assume that for any finite interval $[a, b]$ there exists $p \geq 0$ such that $t \mapsto L_{t} \in \mathcal{Z}_{p}\left((E),(E)^{*}\right)$ is continuous on $[a, b]$. Then $\left\{\Xi_{\Delta}\right\} \subset \mathcal{Z}\left((E),(E)^{*}\right)$ is a Cauchy net with respect to the usual order of partitions $\Delta$.

Proof. Note that $t \mapsto L_{t} \in \mathcal{Y}_{p}\left((E),(E)^{*}\right)$ is uniformly continuous on $[a, b]$. Therefore given $\varepsilon>0$ there exists a constant $\gamma>0$ such that

$$
\left\|L_{\mathrm{s}}-L_{t}\right\|_{-p}<\varepsilon, \quad s, t \in[a, b], \quad|s-t|<\gamma .
$$

Let $\Delta^{\prime}$ be a refinement of $\Delta$. Then every small interval $\left[s_{l}, s_{l+1}\right]$ belonging to $\Delta$ is partitioned into a sum of smaller intervals belonging to $\Delta^{\prime}$, say, 


$$
s_{J}=t_{0}^{(\prime)}<t_{1}^{(\prime)}<\cdots<t_{m_{l}}^{(\prime)}=s_{j+1} .
$$

Then, in view of (3.7) we have

$$
\left|\widehat{\Xi_{\Delta^{\prime}}}(\xi, \eta)-\widehat{\Xi_{\Delta}}(\xi, \eta)\right| \leq \sum_{j=0}^{n-1} \sum_{i=0}^{m_{j}-1}\left|\widehat{L_{t_{t}^{(\prime)}}}(\xi, \eta)-\widehat{L_{i}}(\xi, \eta)\right| \int_{t_{t}^{(j)}}^{t_{t^{\prime}+1}^{(\prime)}}|\xi(u)| d u .
$$

Suppose $|\Delta| \equiv \max _{J}\left|s_{J+1}-s_{J}\right|<\gamma$ (then $\left|\Delta^{\prime}\right|<\gamma$ too). Then by (2.7) and (3.8) we come to

$$
\begin{aligned}
\left|\widehat{\Xi_{\Delta^{\prime}}}(\xi, \eta)-\widehat{\Xi_{\Delta}}(\xi, \eta)\right| & \leq \varepsilon \exp \frac{1}{2}\left(|\xi|_{p}^{2}+|\eta|_{p}^{2}\right) \times \int_{a}^{b}|\xi(u)| d u \\
& \leq \varepsilon(b-a)^{1 / 2}|\xi|_{0} \exp \frac{1}{2}\left(|\xi|_{p}^{2}+|\eta|_{p}^{2}\right) .
\end{aligned}
$$

Therefore,

$$
\left|\widehat{\Xi_{\Delta^{\prime}}}(\xi, \eta)-\widehat{\Xi_{\Delta}}(\xi, \eta)\right| \leq \varepsilon \exp K\left(|\xi|_{p}^{2}+|\eta|_{p}^{2}\right),
$$

where

$$
K=\frac{b-a}{2 e} \rho^{2 p}+\frac{1}{2} .
$$

In view of the precise result in [23, Theorem 4.4.7], we conclude from (3.9) that there exist $q>0$ and $M \geq 0$ (independent of the partitions $\Delta$ with $|\Delta|<\gamma$ ) such that

$$
\left\|\left(\Xi_{\Delta^{\prime}}-\Xi_{\Delta}\right) \phi\right\|_{-(p+q+1)} \leq \varepsilon M\|\phi\|_{p+q+1}, \quad \phi \in(E) .
$$

Namely, $\left\{\Xi_{\Delta}\right\}$ is a Cauchy net in $\mathscr{L}\left((E)_{p+q+1},(E)_{-(p+q+1)}\right)$ and therefore in $\mathscr{L}^{\prime}\left((E),(E)^{*}\right)$.

The above lemma says that there exists a unique limit of $\left\{\Xi_{\Delta}\right\} \subset Y((E)$, $\left.(E)^{*}\right)$. The limit is denoted by

$$
\int_{a}^{b} L_{1} d A_{,}=\lim _{\Delta} \sum L_{s_{1}}\left(A_{\imath_{1+1}}-A_{\iota_{1}}\right) .
$$

This is called the quantum stochastic integral of Itô type against the annihilation process and coincides essentially with the one introduced by HudsonParthasarathy [12]. However, only for the existence of the limit we do not need to take $L_{t}$, as a representative value of $L_{t}$ on the interval $\left[s_{1}, s_{1+1}\right]$. Our special choice called Itô type will be important later in $\$ 4$.

Theorem 3.10. Let $\left\{L_{t}\right\}$ be a quantum stochastic process. Assume that for any finite interval $[a, b]$ there exists $p \geq 0$ such that $t \mapsto L_{1} \in \mathcal{Z}_{p}\left((E),(E)^{*}\right)$ is continuous on $[a, b]$. 
Then

$$
\int_{a}^{b} L_{1} d A_{s}=\int_{a}^{b} L_{1} \partial_{1} d s
$$

Proof. For simplicity we denote by $\Xi$ and $\Omega$ the left and right hand sides, respectively. It follows from Lemma 3.9 that

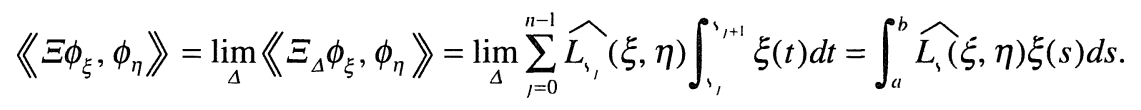

On the other hand, by definition

$$
\left\langle\Omega \phi_{\xi}, \phi_{\eta}\right\rangle=\int_{a}^{b}\left\langle\left\langle L_{1} \partial_{,} \phi_{\xi}, \phi_{\eta}\right\rangle d s=\int_{a}^{b}\left\langle L_{s} \phi_{\xi}, \phi_{\eta}\right\rangle \xi(s) d s .\right.
$$

Therefore $\left\langle\Xi \Xi \phi_{\xi}, \phi_{\eta}\right\rangle=\left\langle\left\langle\Omega \phi_{\xi}, \phi_{\eta}\right\rangle\right.$, which proves the assertion.

qed

Here is a simple example. The number process is expressed as

$$
\Lambda_{t}=\int_{0}^{t} \partial_{,}^{*} \partial_{,} d s=\int_{0}^{t} \partial_{,}^{*} d A_{,}
$$

In a similar manner, for a quantum stochastic process $\left\{L_{t}\right\}$ satisfying the same condition as stated in Theorem 3.10, one may prove the existence of limit:

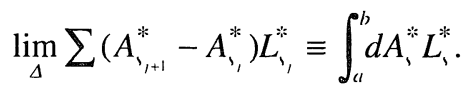

Here again we adopt the Riemannian sum of Itô type, though it is not necessary just for ensuring the existence of the limit. The next result is straightforward from the above argument and (3.4).

Proposition 3.11. For a quantum stochastic process $\left\{L_{t}\right\}$ satisfying the same condition as stated in Theorem 3.10, we have

$$
\int_{a}^{b} d A_{s}^{*} L_{s}^{*}=\left(\int_{a}^{b} L_{1} d A_{1}\right)^{*}=\int_{a}^{b} \partial^{*} L_{1}^{*} d s .
$$

\section{§. Adapted Processes}

\section{§4.1. Definition}

As introduced in $\S 1.3$ we put

$$
D_{1}=\Xi_{0,1}(y)=\int_{R} y(t) \partial_{t} d t, \quad D_{1}^{*}=\Xi_{1,0}(y)=\int_{R} y(t) \partial_{t}^{*} d t, \quad y \in E_{C}^{*} .
$$


It follows from Theorem 1.6 that for $y=\eta \in E_{C}, D_{\eta}^{*} \in \mathscr{L}((E),(E))$ and $D_{\eta}$ is continuously extended to an operator from $(E)^{*}$ into itself, i.e., $D_{\eta} \in \mathscr{L}\left((E)^{*}\right.$, $\left.(E)^{*}\right)$. Thus for $\Xi \in \mathscr{L}\left((E),(E)^{*}\right)$ the commutators $\left[D_{\eta}, \Xi\right]$ and $\left[D_{\eta}^{*}, \Xi\right]$ are meaningful.

Motivated by Hudson-Parthasarathy [12], we make the following

Definition 4.1. A generalized quantum stochastic process $\Xi \in \mathscr{L}\left(E_{C}\right.$, $\mathscr{L}\left((E),(E)^{*}\right)$ ) is called adapted (with respect to the filtration generated by $\left.\left\{\partial_{t}, \partial_{t}^{*}\right\}\right)$ if

$$
\left[D_{\eta}, \Xi(\xi)\right]=\left[D_{\eta}^{*}, \Xi(\xi)\right]=0
$$

for any choice of $t \in \mathbb{R}, \xi, \eta \in E_{C}$ such that supp $\xi \subset(-\infty, t)$ and supp $\eta \in(t,+\infty)$.

Lemma 4.2. Assume that a generalized quantum stochastic process $\Xi$ is regular. Then it is adapted if and only if $\left[D_{\eta}, \Xi_{t}\right]=\left[D_{\eta}^{*}, \Xi_{t}\right]=0$ for any $t \in \mathbb{R}$ and $\eta \in E_{C}$ with supp $\eta \subset(t,+\infty)$.

Proof. First we assume that $\Xi$ is adapted. Given $t \in \mathbb{R}$ and $\eta \in E_{C}$ with supp $\eta \subset(t,+\infty)$, take $s<t$ and a $\delta$-sequence $\xi_{n} \in E_{C}$ such that supp $\xi_{n} \subset(-\infty, t)$ and $\xi_{n} \rightarrow \delta_{1}$. Then, since $\Xi\left(\xi_{n}\right) \rightarrow \Xi\left(\delta_{1}\right)=\Xi_{1}$, we have

$$
\left[D_{\eta}, \Xi_{1}\right]=\lim _{n \rightarrow \infty}\left[D_{\eta}, \Xi\left(\xi_{n}\right)\right]=0
$$

and hence $\left[D_{\eta}, \Xi_{1}\right]=0$ for any $s<t$. Letting $s \rightarrow t$, we obtain $\left[D_{\eta}, \Xi_{t}\right]=0$ as desired.

We prove the converse. Given $\xi, \eta \in E_{C}$ such that supp $\xi \subset(-\infty, t)$ and supp $\eta \subset(t,+\infty)$ take $t_{1}<t$ as supp $\xi \subset\left(-\infty, t_{1}\right)$. Then by Lemma 2.3,

$$
\left.\| D_{\eta} \Xi(\xi) \phi, \psi\right\rangle=\left\langle\left\langle\Xi(\xi) \phi, D_{\eta}^{*} \psi\right\rangle=\int_{-\infty}^{t_{1}} \xi(s) 《\left\langle\Xi, \phi, D_{\eta}^{*} \psi\right\rangle d s .\right.
$$

On the other hand, we have by assumption

$$
\left\langle\Xi \Xi_{1} \phi, D_{\eta}^{*} \psi\right\rangle=\left\langle\left\langle D_{\eta} \Xi, \phi, \psi\right\rangle=\left\langle\left\langle\Xi, D_{\eta} \phi, \psi\right\rangle, \quad s \leq t_{1} .\right.\right.
$$

Hence (4.1) becomes

$$
\int_{-\infty}^{t_{1}} \xi(s) 《\left\langle\Xi, D_{\eta} \phi, \psi\right\rangle d s=\left\langle\left\langle\Xi(\xi) D_{\eta} \phi, \psi\right\rangle .\right.
$$

Consequently, it follows from (4.1) and (4.2) that $D_{\eta} \Xi(\xi)=\Xi(\xi) D_{\eta}$.

Taking the above result into account, we make the following definition which is consistent with Huang's one [11].

Definition 4.3. A quantum stochastic process $\left\{\Xi_{1}\right\}$ is called adapted if 


$$
\left[D_{\eta}, \Xi_{t}\right]=\left[D_{\eta}^{*}, \Xi_{t}\right]=0
$$

for any $t \in \mathbb{R}$ and $\eta \in E_{C}$ with supp $\eta \subset(t,+\infty)$.

\section{\$4.2. Fock Expansion of Adapted Processes}

We first study operators commuting with $D_{\eta}$. By a straightforward computation using the canonical commutation relation we obtain

$$
\left[D_{\eta}, \Xi_{l, m}\left(\kappa_{l, m}\right)\right]=l \Xi_{l-1, m}\left(\kappa_{l, m} \otimes^{1} \eta\right), \quad \eta \in E_{C}, \quad \kappa_{l, m} \in\left(E_{C}^{\otimes(l+m)}\right)_{\mathrm{sym}(l, m)}^{*} .
$$

(See also [25].)

Lemma 4.4. Let $\Xi \in \mathcal{L}^{\prime}\left((E),(E)^{*}\right)$ and let

$$
\Xi=\sum_{l, m=0}^{\infty} \Xi_{l, m}\left(\kappa_{l, m}\right), \quad \kappa_{l, m} \in\left(E_{C}^{\otimes(l+m)}\right)_{\mathrm{sym}(l, m)}^{*},
$$

be the Fock expansion. Given $\eta \in E_{C},\left[D_{\eta}, \Xi\right]=0$ if and only if $\kappa_{l, m} \otimes^{1} \eta=0$ for all $l \geq 1$ and $m \geq 0$.

Proof. In view of (4.3) we obtain

$$
\left[D_{\eta}, \Xi\right]=\sum_{l, m=0}^{\infty} l \Xi_{l-1, m}\left(\kappa_{l, m} \otimes^{1} \eta\right)
$$

which is the Fock expansion of $\left[D_{\eta}, \Xi\right]$. Because of the uniqueness of the Fock expansion $\left[D_{\eta}, \Xi\right]=0$ if and only if $\Xi_{l-1, m}\left(\kappa_{l, m} \otimes^{1} \eta\right)=0$ for all $l \geq 1$ and $m \geq 0$; and therefore, if and only if $\kappa_{l, m} \otimes^{1} \eta=0$ for all $l \geq 1$ and $m \geq 0$.

qed

The support of a distribution $\kappa \in\left(E_{C}^{\otimes_{n}}\right)^{*}$, denoted by supp $\kappa$, is the smallest closed subset $F \subset \mathbb{R}^{n}$ such that $\kappa$ vanishes in $\mathbb{R}^{n}-F$. Therefore supp $\kappa \subset F, F$ being a closed subset of $\mathbb{R}^{n}$, if and only if $\kappa$ vanishes outside $F$.

Lemma 4.5. Let $\Xi \in \mathscr{L}\left((E),(E)^{*}\right)$ with the Fock expansion given as in (4.4). Let $t \in \mathbb{R}$ be fixed. Then $\left[D_{\eta}, \Xi\right]=0$ for any $\eta \in E_{C}$ with supp $\eta \subset(t,+\infty)$ if and only if supp $\kappa_{l, m} \subset(-\infty, t]^{l} \times \mathbb{R}^{m}$ for all $l, m$ with $l+m \geq 1$.

Proof. By Lemma 4.4, $\left[D_{\eta}, \Xi\right]=0$ for any $\eta \in E_{C}$ with supp $\eta \subset(t,+\infty)$ if and only if

$$
\left\langle\kappa_{l, m} \otimes^{\prime} \eta, \eta_{1}^{\otimes(l-1)} \otimes \xi_{1}^{\otimes m}\right\rangle=\left\langle\kappa_{l, m} \otimes_{m} \xi_{1}^{\otimes m}, \eta \otimes \eta_{1}^{\otimes(l-1)}\right\rangle=0
$$

for any $\xi_{1}, \eta_{1} \in E_{C}$ and $\eta \in E_{C}$ with supp $\eta \subset(t,+\infty)$. Therefore $\kappa_{l, m} \otimes_{m} \xi_{1}^{\otimes m}$ vanishes outside $(-\infty, t] \times \mathbb{R}^{l-1}$. Since $\kappa_{l, m} \otimes_{m} \xi_{1}^{\otimes_{m}}$ is symmetric by assumption, it 
vanishes outside $(-\infty, t]^{l}$. Consequently, $\kappa_{l, m}$ vanishes outside $(-\infty, t]^{l} \times \boldsymbol{R}^{m}$. The converse assertion is clear.

Lemma 4.6. Let $\Xi \in \mathscr{L}\left((E),(E)^{*}\right)$ and let $t \in \mathbb{R}$ be fixed. Then $\left[D_{\eta}, \Xi\right]=$ $\left[D_{\eta}^{*}, \Xi\right]=0$ for any $\eta \in E_{C}$ with supp $\eta \subset(t,+\infty)$ if and only if the Fock expansion of $\Xi$ is of the form:

$$
\Xi=\sum_{l+m \geq 1} \Xi_{l, m}\left(\kappa_{l, m}\right)+c I, \quad c=《 \Xi \phi_{0}, \phi_{0} 》,
$$

where supp $\kappa_{l, m} \subset(-\infty, t]^{l+m}$ for all $l, m \geq 0$ with $l+m \geq 1$.

Proof. Note first that $\left[D_{\eta}^{*}, \Xi\right]=0$ if and only if $\left[D_{\eta}, \Xi^{*}\right]=0$. Then the assertion follows from Lemma 4.5 .

Combining Proposition 2.5 and Lemma 4.6 above, we obtain

Theorem 4.7. Let $\Xi$ be a generalized quantum stochastic process and let

$$
\Xi(\xi)=\sum_{l+m \geq 1} \Xi_{l, m}\left(\kappa_{l, m}(\xi)\right)+\langle c, \xi\rangle I, \quad \xi \in E_{\boldsymbol{C}},
$$

be the Fock expansion, where $\kappa_{l, m} \in \mathcal{Z}\left(E_{C},\left(E_{C}^{\otimes(l+m)}\right)_{\mathrm{hym}(l, m)}^{*}\right)$ and $c \in E_{C}^{*}$. Then $\Xi$ is adapted if and only if supp $\kappa_{l, m}(\xi) \subset(-\infty, t]^{l+m}$ for any choice of $t \in \mathbb{R}, \xi \in E_{C}$ with supp $\xi \subset(-\infty, t)$ and $l+m \geq 1$.

Similarly, by Proposition 2.7 and Lemma 4.6 we obtain

Theorem 4.8. Let $\left\{\Xi_{t}\right\}$ be a quantum stochastic process and let

$$
\Xi_{t}=\sum_{l+m \geq 1} \Xi_{l, m}\left(\kappa_{l, m}(t)\right)+c_{t} I, \quad t \in \mathbb{R},
$$

be the Fock expansion. Then $\left\{\Xi_{t}\right\}$ is adapted if and only if supp $\kappa_{l, m}(t) \subset$ $(-\infty, t]^{l+m}$ for any $t \in \mathbb{R}$ and $l+m \geq 1$.

The annihilation and creation processes:

$$
A_{t}=\int_{-\infty}^{t} \partial_{,} d s=\Xi_{0,1}\left(1_{(-\infty, t]}\right), \quad A_{t}^{*}=\int_{-\infty}^{t} \partial_{i}^{*} d s=\Xi_{1,0}\left(1_{(-\infty, t]}\right), \quad t \in \mathbb{R},
$$

defined as integral kernel operators are adapted. Similarly, the number process

$$
\Lambda_{t}=\int_{-\infty}^{t} \partial_{1}^{*} \partial_{1} d s=\Xi_{1,1}\left(\tau_{(-\infty, t]}\right), \quad t \in \boldsymbol{R},
$$

is adapted. It is also obvious that $\left\{\partial_{t}\right\},\left\{\partial_{t}^{*}\right\}$ and $\left\{\partial_{t}^{*} \partial_{t}\right\}$ are adapted. 


\section{\$4.3. Stochastic Integral Representation}

In $\$ 2.4$ we established a stochastic integral-like representation for a quantum stochastic process. We now consider the case of adapted processes.

Theorem 4.9. Assume that a generalized quantum stochastic process $\Xi$ is adapted. Then it is expressed as

$$
\Xi(\xi)=\int_{R} L(\xi, s) \partial_{s} d s+\int_{R} \partial^{*} M^{*}(\xi, s) d s+\langle c, \xi\rangle I, \quad \xi \in E_{C},
$$

where

(i) $L \in \mathscr{B}\left(E_{C}, E_{C} ; \mathscr{L}^{\prime}\left((E),(E)^{*}\right)\right)$ such that $\operatorname{supp} L(\xi, \cdot) \subset(-\infty, t]$ for any $\xi \in E_{C}$ with $\operatorname{supp} \xi \subset(-\infty, t)$;

(ii) $M \in \mathscr{B}\left(E_{C}, E_{C} ; \mathscr{L}^{\prime}((E),(E))\right)$ such that $\left[M(\xi, \eta), \partial_{t}\right]=0$ for any $\xi, \eta \in E_{C}$, $t \in \boldsymbol{R}$ and $\operatorname{supp} M(\xi, \cdot) \subset(-\infty, t]$ for any $\xi \in E_{C}$ with supp $\xi \subset(-\infty, t)$;

(iii) $c \in E_{C}^{*}$.

To see the above result we only need to follow the proof of Theorem 2.8 keeping Theorem 4.7 into account. If the generalized quantum stochastic process $\Xi$ is regular, combination with Theorem 2.12 yields

Theorem 4.10. Let $\Xi$ be an adapted generalized quantum stochastic process and consider the expression as in Theorem 4.9. If $\Xi$ is regular, then $L \in \cdot \mathscr{K}_{\text {sep }}\left(E_{C}^{*}, E_{C} ; \mathcal{I}\left((E),(E)^{*}\right)\right), M \in \cdot \mathscr{M}_{\text {iep }}\left(E_{C}^{*}, E_{C} ; \mathcal{Y}((E),(E))\right)$ and $c \in E_{C}$.

Recall that the delta functions $\delta_{1}$, belong to $E_{-p}$ for $p>5 / 12$. On the other hand, the separately continuous bilinear maps $L$ and $M$ in the above theorem become jointly continuous on $E_{C .-p} \times E_{C}$. We thus come to

Corollary 4.11. Let $\Xi$ be a regular generalized quantum stochastic process. If it is adapted, it is expressed as

$$
\Xi_{t}=\int_{-\infty}^{t} L(t, s) \partial_{s} d s+\int_{-\infty}^{t} \partial_{i}^{*} M^{*}(t, s) d s+c_{t} I, \quad t \in \boldsymbol{R},
$$

where

(i) $L: \boldsymbol{R} \times E_{C} \rightarrow \mathcal{Z}^{\prime}\left((E),(E)^{*}\right)$ is continuous; linear in the second argument; and supp $L(t, \cdot) \subset(-\infty, t]$ for all $t \in \mathbb{R}$;

(ii) $M: \mathbb{R} \times E_{C} \rightarrow \mathscr{Z}((E),(E))$ is continuous; linear in the second argument; $\left[M(s, \xi), \partial_{t}\right]=0$ for any $\xi \in E_{C}$ and $s, t \in \mathbb{R}$; and $\operatorname{supp} M(t, \cdot) \subset(-\infty, t]$ for all $t \in \boldsymbol{R}$

(iii) $c: \boldsymbol{R} \rightarrow \boldsymbol{C}$ is a continuous function. 


\section{§4.4. Quantum Stochastic Imtegrals off Hî̀ Type}

There is a close connection between quantum stochastic integrals of Itô type [12] and our integrals. In case of an integral against the annihilation process the result is already stated in Theorem 3.10 without assuming the adaptedness.

Lemma 4.12。 Let $\left\{L_{t}\right\} \subset \mathscr{L}^{\prime}((E),(E))$ be an adapted quantum stochastic process. Then,

$$
\left[L_{t}, A_{t+h}-A_{t}\right]=0, \quad t \in \mathbb{R}, \quad h \geq 0 .
$$

Proof. By definition $\left[D_{\eta}, L_{t}\right]=\left[D_{\eta}^{*}, L_{t}\right]=0$ for any $\eta \in E_{C}$ with supp $\eta \subset(t,+\infty)$. Since $L_{t} \in \mathscr{L}^{\prime}((E),(E))$, for any $y \in E_{C}^{*}$ the commutator $\left[D_{1}, L_{t}\right]$ is well-defined and belongs to $\mathscr{Z}((E),(E))$. Put $y=1_{[t, t+h]}$ and take an approximating sequence $\eta_{n} \in E_{C}$ with supp $\eta_{n} \subset(t,+\infty)$ such that $\eta_{n} \rightarrow y$ in $E_{C}^{*}$. Since $D_{1}=A_{t+h}-A_{t}$, we have

$$
\left[L_{t}, A_{t+h}-A_{t}\right]=\left[L_{t}, D_{1}\right]=\lim _{n \rightarrow \infty}\left[L_{t}, D_{\eta_{n}}\right]=0,
$$

as desired.

qed

Lemima 4.13。 Let $\left\{L_{t}\right\} \subset \mathcal{Z}((E),(E))$ be an adapted quantum stochastic process. Assume that for any finite interval $[a, b]$ there exists $p \geq 0$ such that $t \mapsto L_{t} \in \mathscr{Z}_{p}\left((E),(E)^{*}\right)$ is continuous on $[a, b]$. Then the limit

$$
\int_{a}^{b} L_{s}^{*} d A_{s}^{*} \equiv \lim _{\Delta} \sum L_{s_{1}}^{*}\left(A_{s_{1+1}}^{*}-A_{s_{s}}^{*}\right)
$$

exists in $\mathcal{L}\left((E),(E)^{*}\right)$. Moreover

$$
\int_{a}^{b} L_{s} d A_{s}=\left(\int_{a}^{b} L_{s} d A_{s}\right)^{*}
$$

Proof. By Lemma 4.12 we have

$$
\sum\left(A_{s_{1+1}}-A_{s_{1}}\right) L_{r_{1}}=\sum L_{s_{1}}\left(A_{s_{1+1}}-A_{r_{1}}\right) .
$$

Then, apply Lemma 3.9 to observe that

$$
\lim _{\Delta} \sum\left(A_{s_{i+1}}-A_{s_{1}}\right) L_{r_{1}}=\int_{a}^{b} L_{i} d A_{i} .
$$

The assertion follows by taking the adjoint.

qed

The stochastic integral defined in (4.7) is essentially the same as one due to Hudson-Parthasarathy [12]. The next result is clear for Proposition 3.11. 
Theorem 4.14. Let $\left\{L_{t}\right\} \subset \mathscr{L}((E),(E))$ be an adapted quantum stochastic process. Assume that for any finite interval $[a, b]$ there exists $p \geq 0$ such that $t \mapsto L_{t} \in \mathscr{L}_{p}^{\prime}\left((E),(E)^{*}\right)$ is continuous on $[a, b]$. Then

$$
\int_{a}^{b} L_{s}^{*} d A_{s}^{*}=\int_{a}^{b} \partial^{*} L_{i}^{*} d s .
$$

\section{\$4.5. Invariance of Operator Symbols}

It is possible to describe the adaptedness of a quantum stochastic process in terms of operator symbols. We here prove the following

Theorem 4.15. Let $\left\{\Xi_{t}\right\}$ be a quantum stochastic process. Then it is adapted if and only if the function

$$
(\xi, \eta) \mapsto e^{-\langle\xi, \eta\rangle} \hat{\Xi}_{t}(\xi, \eta), \quad \xi, \eta \in E_{C},
$$

is invariant under the translations: $\xi \rightarrow \xi+\xi_{1}$ and $\eta \rightarrow \eta+\eta_{1}$ where $\xi_{1}, \eta_{1} \in E_{C}$ with supp $\xi_{1}$, supp $\eta_{1} \subset(t,+\infty)$.

Proof. Consider the Fock expansion:

$$
\Xi_{t}=\sum_{I+m \geq 1} \Xi_{l, m}\left(\kappa_{l, m}(t)\right)+c_{t} I
$$

Then

$$
\Psi(\xi, \eta) \equiv e^{-\langle\xi, \eta\rangle} \hat{\Xi}_{t}(\xi, \eta)=\sum_{I+m \geq 1}\left\langle\kappa_{l, m}(t), \eta^{\otimes I} \otimes \xi^{\otimes m}\right\rangle+c_{t}, \quad \xi, \eta \in E_{C} .
$$

If $\Xi_{t}$ is adapted, it follows from Theorem 4.8 that $\operatorname{supp} \kappa_{l, m}(t) \subset(-\infty, t]^{l+m}$. Hence for any $\xi_{1}, \eta_{1} \in E_{C}$ with $\operatorname{supp} \xi_{1}, \quad \operatorname{supp} \eta_{1} \subset(t,+\infty)$ we have $\Psi(\xi, \eta)=$ $\Psi\left(\xi+\xi_{1}, \eta+\eta_{1}\right)$.

Conversely, we assume the invariance property of $\Psi$. Then, in particular,

$$
\sum_{I+m \geq 1}\left\langle\kappa_{l, m}(t), \eta_{1}^{\otimes I} \otimes \xi_{l}^{\otimes m}\right\rangle=0
$$

for any $\xi_{1}, \eta_{1} \in E_{C}$ with supp $\xi_{1}$, supp $\eta_{1} \subset(t,+\infty)$. Hence supp $\kappa_{l, m}(t) \subset(-\infty, t]^{l+m}$ and $\Xi_{t}$ is adapted.

qed

\section{Appendices: Norm Inequalities}

\section{§.1. Integral Kermel Operators}

For $\kappa \in\left(E_{C}^{\otimes(I+m)}\right)^{*}$ we put

$$
|\kappa|_{l, m, p, q}=\left|\left(\left(A^{\otimes l}\right)^{p} \otimes\left(A^{\otimes m}\right)^{q}\right) \kappa\right|_{0}, \quad p, q \in \mathbb{R} .
$$

Obviously, $|\kappa|_{-p}=|\kappa|_{\ell, m,-p,-p}$ which is finite for all sufficiently large $p \geq 0$. 
Theorem $\mathbb{A} .1$ ([23, Theorem 4.3.9])。 Let $\kappa \in\left(E_{C}^{\otimes(l+m)}\right)^{*}$. Then for any $p \in \mathbb{R}, q>0$ and $\alpha, \beta>0$ with $\alpha+\beta \leq 2 q$, it holds that

$$
\left\|\Xi_{l, m}(\kappa) \phi\right\|_{p} \leq \rho^{-q / 2}\left(l^{\prime} m^{m}\right)^{1 / 2}\left(\frac{\rho^{-\alpha / 2}}{-\alpha e \log \rho}\right)^{1 / 2}\left(\frac{\rho^{-\beta / 2}}{-\beta e \log \rho}\right)^{m / 2}|\kappa|_{I, m, p,-(p+q)}\|\phi\|_{p+q},
$$

for all $\phi \in(E)$.

It is known that $\kappa \in\left(E_{C}^{\otimes(I+m)}\right)^{*}$ belongs to the smaller subspace $\left(E_{C}^{\otimes l}\right) \otimes\left(E_{C}^{\otimes m}\right)^{*}$ if and only if for any $p \geq 0$ there exists $q \geq 0$ such that $|\kappa|_{l, m ; p,-(p+q)}<\infty$. Specializing parameters in Theorem A.1, we obtain

Lemma A.2. Let $\kappa \in\left(E_{C}^{\otimes(l+m)}\right)^{*}$. Then, for any $p>0$ with $|\kappa|_{-p}<\infty$ we have

$$
\left\|\Xi_{l, m}(\kappa) \phi\right\|_{-p} \leq C_{l, m, p}|\kappa|_{-p}\|\phi\|_{p}, \quad \phi \in(E)
$$

where

$$
C_{l, m, p}=\rho^{-p}\left(l^{l} m^{m}\right)^{1 / 2}\left(\frac{\rho^{-p}}{-2 p e \log \rho}\right)^{(l+m) / 2} .
$$

Lemma A.3. Let $\kappa \in\left(E_{C}^{\otimes m}\right)^{*}$. Then for any $p \in \mathbb{R}$ and $q>0$ with $|\kappa|_{-(p+q)}$ $<\infty$ we have

$$
\left\|\Xi_{0 . m}(\kappa) \phi\right\|_{p} \leq C_{m . q}|\kappa|_{-(p+q)}\|\phi\|_{p+q}, \quad \phi \in(E)
$$

where

$$
C_{m, q}=\rho^{-q / 2} m^{m / 2}\left(\frac{\rho^{-q}}{-2 q e \log \rho}\right)^{m / 2}
$$

\section{§.2. Delta Functions}

Theorem $\mathbb{A}$.4. For any $p>5 / 12$ and $0 \leq \alpha \leq 1$ with $p-5 / 12>\alpha / 2$ there exists a constant number $C=C(p, \alpha) \geq 0$ such that

$$
\left|\delta_{1}-\delta_{t}\right|_{-p} \leq C|s-t|^{\alpha}, \quad s, t \in \mathbb{R} .
$$

In particular, $t \mapsto \delta_{t} \in E_{-p}$ is continuous for $p>5 / 12$.

Proof. For each $j=0,1,2, \ldots$ we put

$$
e_{j}(t)=\left(\sqrt{\pi} 2^{\jmath} j !\right)^{-1 / 2} e^{-t^{2} / 2} H_{j}(t),
$$

where $H_{J}$ is the Hermite polynomial of degree $j$, see [23], [29]. Then $\left\{e_{J}\right\}_{j=0}^{\infty}$ is a complete orthonormal basis of $L^{2}(\mathbb{R}, d t)$. Note also that 


$$
A e_{J}=(2 j+2) e_{,}, \quad A=1+t^{2}-\frac{d^{2}}{d t^{2}} .
$$

Then by definition

$$
|f|_{-p}^{2}=\left|A^{-p} f\right|_{0}^{2}=\sum_{j=0}^{\infty}\left|\left\langle f, e_{J}\right\rangle\right|^{2}(2 j+2)^{-2 p}, \quad f \in E_{C}^{*}, \quad p \in \boldsymbol{R} .
$$

In particular, for $s, t \in \boldsymbol{R}$,

$$
\text { (A.2) } \begin{aligned}
\left|\delta_{1}-\delta_{t}\right|_{-p}^{2} & =\sum_{j=0}^{\infty}\left|\left\langle\delta_{i}-\delta_{t}, e_{J}\right\rangle\right|^{2}(2 j+2)^{-2 p} \\
& =\sum_{j=0}^{\infty}\left|e_{l}(s)-e_{l}(t)\right|^{2}(2 j+2)^{-2 p} \\
& \leq \sum_{j=0}^{\infty}|s-t|^{2 \alpha} \sup _{u \in R}\left|e_{j}^{\prime}(u)\right|^{2 \alpha} \times 2^{2-2 \alpha} \sup _{u \in R}\left|e_{j}(u)\right|^{2-2 \alpha} \times(2 j+2)^{-2 p} .
\end{aligned}
$$

Since $e_{l}^{\prime}=\sqrt{j / 2} e_{J-1}-\sqrt{(j+1) / 2} e_{\jmath+1}$, which is verified by a direct computation, we have

$$
\left|e_{,}^{\prime}(u)\right|_{1}^{2 \alpha} \leq j^{\alpha} \gamma_{1-1}^{2 \alpha}+(j+1)^{\alpha} \gamma_{1+1}^{2 \alpha}, \quad u \in \boldsymbol{R}
$$

where

$$
\gamma_{1}=\sup _{u \in R}\left|e_{1}(u)\right|, \quad j=0,1,2, \cdots
$$

Then (A.2) becomes

$$
\left|\delta_{,}-\delta_{t}\right|_{-p}^{2} \leq 2^{2-2 \alpha}|s-t|^{2 \alpha} \sum_{l=0}^{\infty}\left(j^{\alpha} \gamma_{l-1}^{2 \alpha}+(j+1)^{\alpha} \gamma_{l+\mathrm{i}}^{2 \alpha}\right) \gamma_{l}^{2-2 \alpha}(2 j+2)^{-2 p} .
$$

Now we recall Szegö's theorem $[29,8.91 .10]: \lim _{1 \rightarrow \infty} j^{1 / 12} \gamma$, exists and is finite. Thereby the series in (A.3) converges for $\alpha-1 / 6-2 p<-1$ and we obtain (A.1).

\section{References}

[1] Belavkin, V. P., A quantum nonadapted Itô formula and stochastic analysis in Fock scale, $J$. Funct. Anal., 102 (1991), 414-447.

[2] , Quantum stochastic calculus and quantum nonlinear filtering, J. Multivariate Anal., 42 (1992), 171-201.

[3] - Chaotic states and stochastic integration in quantum systems, Russian Math. Surveys, 47 (1992), 53-116.

[4] Berezin, F. A., The Method of Second Quantization, Academic Press, 1966.

[5] Bogolubov, N. N., Logunov, A. A. and Todorov, I. T., Introduction to Axiomatic Quantum Field Theory, Benjamin, Massachusetts, 1975.

[6] Gelfand, I. M. and Vilenkin, N. Ya., Generalized Functions, Vol. 4. Academıc Press, New York, 1964.

[7] Hida, T., Analysis of Brownian Functionals, Carleton Math. Lect. Notes, 13, Carleton University, Ottawa, 1975.

[8] Brownian Motion, Springer-Verlag, 1980.

[9] Hida, T., Kuo, H.-H., Potthoff, J. and Streit, L., White Noise, Kluwer Academic, 1993. 
[10] Hida, T., Obata, N. and Saitô, K., Infinite dimensional rotations and Laplacians in terms of white noise calculus, Nogoya Math. J., 128 (1992), 65-93.

[11] Huang, Z., Quantum white noises-White noise approach to quantum stochastic calculus, Nagoya Math. J., 129 (1993), 23-42.

[12] Hudson, R. L. and Parthasarathy, K. R., Quantum Itô's formula and stochastic evolutions, Commun. Math. Phys., 93 (1984), 301-323.

[13] Krée, P., La théorie des distributions en dimension quelconque et l'intégration stochastique, in Stochastic Analysis and Related Topics, (H. Korezlioglu and A. S. Ustunel, eds.), Lect. Notes in Math., Springer-Verlag, 1316 (1988), 170-233.

[14] Krée, P. and Rączka. R., Kernels and symbols of operators in quantum field theory, Ann. Inst. H. Poincaré Sect., A28 (1978), 41-73.

[15] Kubo, I. and Takenaka, S., Calculus on Gaussian white noise I-IV, Proc. Japan Acad., 56A (1980), 376-380, 411-416; 57A (1981), 433-437; 58A (1982), 186-189.

[16] Lindsay, J. M., On set convolutions and integral-sum kernel operators, in Probability Theory and Mathematical Statistics, Vol. 2 (B. Grigelionis et al. eds.), Mokslas, Vilnius, (1990), 105-123.

[17] - Quantum and non-causal stochastic calculus, Probab. Theory Relat. Fields, 97 (1993), 65-80.

[18] Lindsay, J. M. and Maassen, H., An integral kernel approach to noise, in Quantum Probability and Applications III (L. Accardi and W. von Waldenfels eds.), Lect. Notes in Math., SpringerVerlag, $\mathbb{1 3 0 3}$ (1988), 192-208.

[19] Maassen, H., Quantum Markov processes on Fock space described by integral kernels, in Quantum Probability and Applications II (L. Accardi and W. von Waldenfels eds.), Lect. Notes in Math., Springer-Verlag, 1136 (1985), 361-374.

[20] Meyer, P. A., Elements de probabilités quantiques VI, in Séminaire de Probabilités XXI (J.Azéma et al. eds.), Lect. Notes in Math., Springer-Verlag, 1247 (1987), 34-49.

[21] Quantum Probability for Probabilists, Lect. Notes in Math., Springer-Verlag, 1538 (1993).

[22] Obata, N., An analytic characterization of symbols of operators on white noise functionals, $J$. Math. Soc. Japan, 45 (1993), 421-445.

[23] - White Noise Calculus and Fock Space, Lect. Notes in Math., Springer-Verlag, $\mathbb{1 5 7 7}$ (1994).

[24] Operator calculus on vector-valued white noise functionals, J. Funct. Anal.. $\mathbb{1} 2 \mathbb{1}$ (1994), 185-232.

[25] - Integral kernel operators on Fock space - Generalizations and applications to quantum dynamics, to appear in Acta Appl. Math.

[26] Parthasarathy, K. R., An Introduction to Quantum Stochastic Calculus, Birkhäuser, 1992.

[27] Parthasarathy, K. R. and Sinha, K. B., Stochastic integral representation of bounded quantum martingales in Fock space, J. Funct. Anal., 67 (1986), 126-151.

[28] Schaefer, H. H., Topological Vector Spaces, Springer-Verlag, 1986.

[29] Szegö, G., Orthogonal Polynomials, Amer. Math. Soc., Providence, 1975. 